\title{
Functional impact of Aurora A-mediated phosphorylation of HP1 1 at serine 83 during cell cycle progression
}

Adrienne Grzenda', Phoebe Leonard², Seungmae Seo ${ }^{1}$, Angela J Mathison', Guillermo Urrutia', Ezequiel Calvo ${ }^{3}$, Juan lovanna ${ }^{4}$, Raul Urrutia ${ }^{1,5}$ and Gwen Lomberk ${ }^{1,2,5^{*}}$

\begin{abstract}
Background: Previous elegant studies performed in the fission yeast Schizosaccharomyces pombe have identified a requirement for heterochromatin protein 1 (HP1) for spindle pole formation and appropriate cell division. In mammalian cells, HP1y has been implicated in both somatic and germ cell proliferation. High levels of HP1y protein associate with enhanced cell proliferation and oncogenesis, while its genetic inactivation results in meiotic and mitotic failure. However, the regulation of HP1 $y$ by kinases, critical for supporting mitotic progression, remains to be fully characterized.

Results: We report for the first time that during mitotic cell division, HP1 $\gamma$ colocalizes and is phosphorylated at serine $83\left(\mathrm{Ser}^{83}\right.$ ) in $\mathrm{G}_{2} / \mathrm{M}$ phase by Aurora A. Since Aurora A regulates both cell proliferation and mitotic aberrations, we evaluated the role of HP1y in the regulation of these phenomena using siRNA-mediated knockdown, as well as phosphomimetic and nonphosphorylatable site-directed mutants. We found that genetic downregulation of HP1 $\gamma$, which decreases the levels of phosphorylation of HP1 $\gamma$ at $\operatorname{Ser}^{83}$ (P-Ser ${ }^{83}-\mathrm{HP} 1 \mathrm{\gamma}$ ), results in mitotic aberrations that can be rescued by reintroducing wild type HP1 $\gamma$, but not the nonphosphorylatable S83A-HP1 $\gamma$ mutant. In addition, proliferation assays showed that the phosphomimetic S83D-HP1y increases 5-ethynyl-2'-deoxyuridine (EdU) incorporation, whereas the nonphosphorylatable S83A-HP1Y mutant abrogates this effect. Genome-wide expression profiling revealed that the effects of these mutants on mitotic functions are congruently reflected in $G_{2} / M$ gene expression networks in a manner that mimics the on and off states for P-Ser ${ }^{83}-\mathrm{HP} 1 \gamma$.

Conclusions: This is the first description of a mitotic Aurora A-HP1Y pathway, whose integrity is necessary for the execution of proper somatic cell division, providing insight into specific types of posttranslational modifications that associate to distinct functional outcomes of this important chromatin protein.
\end{abstract}

Keywords: Heterochromatin protein 1 (HP1), Mitosis, Aurora kinase, Epigenetics, Spindle pole, Centrosome

\section{Background}

Heterochromatin protein 1 (HP1), the reader of histone $\mathrm{H} 3$ lysine 9 methylation (H3K9me), was originally discovered through studies in Drosophila melanogaster of mosaic gene silencing, known as position effect variegation $[1,2]$. In human and other mammalian cells, the

\footnotetext{
* Correspondence: lomberk.gwen@mayo.edu

'Laboratory of Epigenetics and Chromatin Dynamics, GIH Division,

Department of Medicine, Biochemistry and Molecular Biology, Guggenheim

10, Mayo Clinic, 200 First Street SW, Rochester, MN 55905, USA

${ }^{2}$ Department of Obstetrics and Gynecology, Guggenheim 10, Mayo Clinic,

200 First Street SW, Rochester, MN 55905, USA

Full list of author information is available at the end of the article
}

three mammalian HP1 isoforms, HP1 $\alpha, \mathrm{HP} 1 \beta$ and HP1 1 , have been well-studied for their localization, as well as their roles within the heterochromatic regions that associate with gene silencing. However, subsequent investigations have made it increasingly unmistakable that HP1 proteins not only localize to heterochromatic regions but also euchromatic regions $[3,4]$. These proteins are involved in diverse cellular processes, ranging from chromatin modification and epigenetic gene silencing to replication and DNA repair to nuclear architecture and chromosomal stability [3,4]. Moreover, HP1 proteins respond to a diversity of signaling pathways and acquire

\section{Biomed Central}


various posttranslational modifications, which impact on their function [5-9]. We have previously reported that, during interphase, phosphorylation of $\mathrm{HP} 1 \gamma$ at serine 83 (P$\mathrm{Ser}^{83}-\mathrm{HP} 1 \gamma$ ) via the cAMP-protein kinase A (PKA) pathway upon activation of cell surface receptors relocates this protein to euchromatin, where it plays a role in transcriptional elongation [8]. Thus, it is essential to define HP1-mediated pathways to map useful networks of membrane-to-chromatin signaling cascades for better understanding of the regulation of important cellular processes.

Ample evidence indicates that $\mathrm{HP} 1 \gamma$ is important during both somatic and germ cell proliferation. Indeed, high levels of HP1Y protein associate with enhanced somatic and meiotic cell proliferation [10]. Genetic inactivation of HP1Y results in both meiotic and mitotic failure $[11,12]$. Studies in primordial germ cells demonstrate that loss of HP1 $\gamma$ also reduces their cell number through impaired cell cycle progression [13]. However, the responsible molecular mechanisms that link this vital biological process to the functional regulation of $\mathrm{HP} 1 \gamma$ remain unknown.

Earlier investigations have found that HP1 $\gamma$ is phosphorylated throughout the cell cycle and, in particular, hyperphosphorylated in mitosis [14]. In the current study, we report a novel pathway, whereby HP1 $\gamma$ is regulated by mitotic kinases, in particular, Aurora kinase A, a master regulator of mitotic transitions [15]. We demonstrate that $\mathrm{HP} 1 \gamma$ is phosphorylated at serine $83\left(\mathrm{Ser}^{83}\right)$ in $\mathrm{G}_{2} / \mathrm{M}$ where it colocalizes with Aurora A kinase, and its mitotic targets, cyclin B1, cyclin B2 and cyclin-dependent kinase 1 (CDK1) during cell division. HP1 $\gamma$ is phosphorylated at $\mathrm{Ser}^{83}$ by Aurora $\mathrm{A}$ in vitro and in cells. In addition, siRNA-mediated knockdown of HP1 $\gamma$ leads to a decrease of P-Ser ${ }^{83}-\mathrm{HP} 1 \gamma$ accompanied by mitotic aberrations. Notably, reintroduction of wild type $\mathrm{HP} 1 \gamma$ rescues, to a significant extent, these abnormal mitotic effects, while the nonphosphorylatable S83A-HP1 $\gamma$ mutant is unable to rescue this consequence of HP1Y knockdown. Congruent with these functions, phosphomimetic S83D-HP1 $\gamma$ results in an increase of cell proliferation, whereas the nonphosphorylatable S83A-HP1 $\gamma$ mutant abrogates this effect. In addition, overexpression of either the S83AHP1 $\gamma$ or S83D-HP1 $\gamma$ mutant supports this effect in resultant cell cycle-related gene expression networks. Thus, together, these results reveal that a novel Aurora A-HP1 $\gamma$ pathway targeting $\mathrm{Ser}^{83}$ phosphorylation is necessary for the proper execution of cell division, thereby extending our knowledge of the biochemical and cell biological function of this important chromatin protein.

\section{Results}

\section{HP1 $Y$ is phosphorylated at the $G_{2} / M$ phase of the cell} cycle

We have previously described that $\mathrm{P}-\mathrm{Ser}^{83}-\mathrm{HP} 1 \gamma$ by PKA mediates extracellular signals during interphase [8].
In the current study, we uncover a new Aurora kinase A-mediated pathway that phosphorylates $\mathrm{Ser}^{83}-\mathrm{HP} 1 \gamma$ during mitosis, which is necessary for the proper execution of this process. For this purpose, we initially analyzed the kinetics of phosphorylation in HeLa cells arrested in different phases of the cell cycle. Treatment with roscovitine, a membrane permeable cyclin-dependent kinase (CDK) inhibitor, that arrests cell cycle progression at the $G_{1} / S$ and $G_{2} / M$ checkpoints [16], resulted in dose-

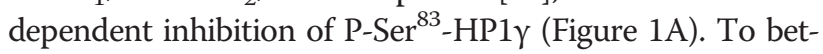
ter define the temporal pattern of these events, we treated with either aphidicolin to arrest cells in $\mathrm{S}$ phase, or nocodazole to obtain mitotic arrest $\left(G_{2} / M\right)$. The mitotic population demonstrated a striking increase in $\mathrm{P}_{-} \mathrm{Ser}^{83}$ HP1Y levels in comparison to the normal cycling population and S phase arrested cells (Figure 1B). To define these events in the absence of kinase inhibitors, we synchronized HeLa cells by double thymidine block to obtain cell extracts at subsequent time points of release from cell cycle arrest. These experiments revealed that the levels of P-Ser ${ }^{83}-\mathrm{HP} 1 \gamma$ peaked twice, the first at 2 hours post-release $\left(\mathrm{G}_{1} /\right.$ $\mathrm{S}$ boundary, Figure 1C, Additional file 1: Figure S1 A). As this peak was likely the phosphorylation event coinciding with the previously described involvement of PKA during interphase [8], we utilized the PKA-specific inhibitor, KT5720, to treat HeLa cells upon release from double thymidine block. Upon KT5720 treatment, P-Ser ${ }^{83}-\mathrm{HP} 1 \gamma$ levels at 2 hours post-release were significantly diminished (Additional file 1: Figure S1 B). However, of greater interest, a more prominent second peak across 8 to 10 hours post-release from cell cycle arrest, which coincided with $\mathrm{G}_{2} / \mathrm{M}$, was observed (Figure $1 \mathrm{C}$, Additional file 1: Figure $\mathrm{S} 1 \mathrm{~A})$. The lower P-Ser ${ }^{83}-\mathrm{HP} 1 \gamma$ levels seen in-between these two peaks (4 to 6 hours post-release, Figure 1C) corresponded with S phase (Additional file 1: Figure S1A), similar to aphidicolin treatment. These results demonstrate that levels of P-Ser ${ }^{83}-\mathrm{HP} 1 \gamma$ peak significantly at $\mathrm{G}_{2} / \mathrm{M}$ phase during the cell cycle, suggesting that phosphorylation of this protein may play a role in cell division.

Subsequently, we sought to complement the biochemical assays of phosphorylation described above by mapping the temporal pattern of staining for $\mathrm{P}-\mathrm{Ser}^{83}-\mathrm{HP} 1 \gamma$ during cell cycle progression. For this purpose, we performed immunofluorescence using confocal microscopy in cells co-stained with the anti-P-Ser ${ }^{83}-\mathrm{HP} 1 \gamma$ and different cell cycle markers. We utilized cyclin D as a marker of $G_{1}$, 5-ethynyl-2'-deoxyuridine (EdU)-pulse labeling for $S$ phase, and cyclin $B$ to indicate the $G_{2}$ and $M$ phases of the cell cycle. Figure 2A,B,C, which represents a low magnification field of cells stained with the anti-P-Ser ${ }^{83}-\mathrm{HP} 1 \gamma$, demonstrates that the level and distribution of the signal for this modified form of $\mathrm{HP} 1 \gamma$ is variable in epithelial cells growing under normal conditions. Thus, we examined more carefully the levels and 


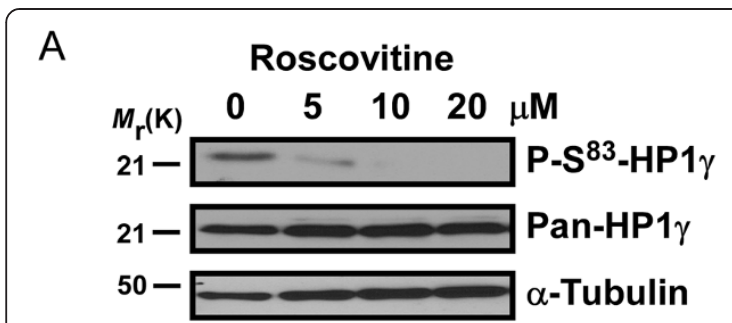

B

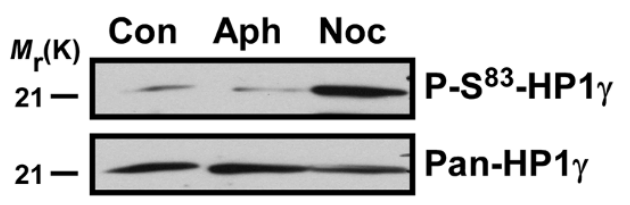

C

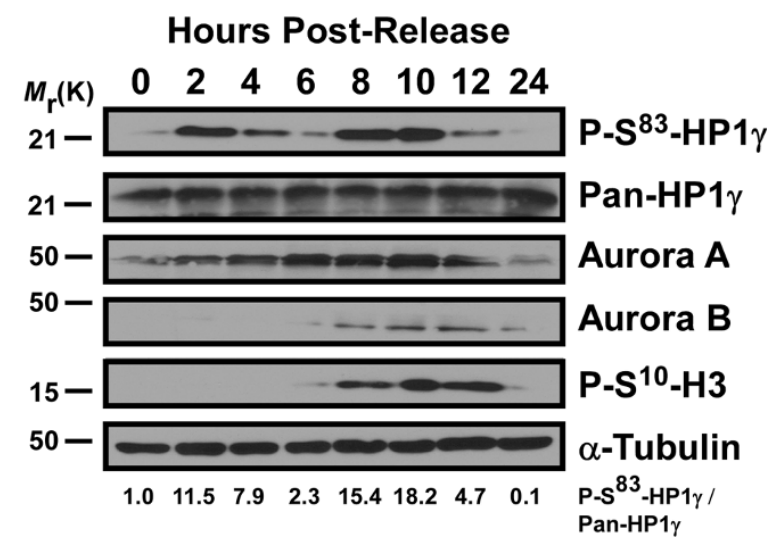

Figure 1 Levels of P-Ser ${ }^{83}$-HP1 1 are cell cycle-dependent, increasing significantly in $\mathbf{G}_{2} / \mathbf{M}$. (A) Inhibition of HP1 $\gamma$

phosphorylation in vivo by the cell cycle inhibitor, roscovitine. HeLa cells incubated with roscovitine, an inhibitor of cell cycle progression at the $G_{1} / S$ and $G_{2} / M$ checkpoints, display a dose-dependent inhibition of phosphorylation as shown by anti-P-Ser ${ }^{83}-\mathrm{HP} 1 \gamma$ (top). atubulin is shown as a loading control (bottom). (B) P-Ser ${ }^{83}-\mathrm{HP} 1 \mathrm{Y}$ levels are high in mitotic arrested cells. Cell extracts were obtained from a normal cycling population (con), cells treated with aphidicolin (aph) to arrest cells in $G_{1} / S$ phase $\left(G_{1} / S\right)$, or mitoticarrested cells $\left(G_{2} / M\right)$ from treatment with nocodazole (noc). An increase of P-Ser ${ }^{83}$-HP1 1 levels in mitosis is shown by comparison of anti-P-Ser ${ }^{83}-\mathrm{HP} 1 \gamma$ (top) with total HP1Y (bottom). (C) P-Ser ${ }^{83}-\mathrm{HP} 1 \gamma$ levels through the cell cycle. HeLa cells were synchronized by double thymidine block and cell extracts were obtained at subsequent time points of release. P-Ser ${ }^{83}-\mathrm{HP} 1 \mathrm{y}$ levels are highest approximately 8 to 10 hours post-release, which corresponds to an increase in the presence of other mitotic markers, including P-Ser ${ }^{10}$. $\mathrm{H} 3$, Aurora A and Aurora B, indicating M phase entry. The relative intensity indicated below was calculated as P-Ser ${ }^{83}-\mathrm{HP} 1 \gamma /$ pan-HP1Y ratios and normalization with the ratio of 0 hour. aph, aphidicolin; con, control; noc, nocodazole; $\mathrm{P}-\mathrm{Ser}^{10}-\mathrm{H} 3$, phosphorylation of histone $\mathrm{H} 3$ at serine 10; $\mathrm{P}-\mathrm{Ser}^{83}-\mathrm{HP} 1 \gamma$, phosphorylation of HP1 1 at serine 83. distribution of $\mathrm{P}-\mathrm{Ser}^{83}-\mathrm{HP} 1 \gamma$ signals in relationship to

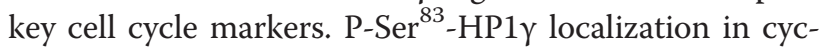
lin D-positive cells $\left(G_{1}\right)$ appeared in the euchromatic compartment of the nucleus as a fine punctate pattern (Figure 2D,E,F). Quantification of cyclin D-positive cells demonstrated that $76.6 \%$ of this population (160/209) had $\mathrm{P}-\mathrm{Ser}^{83}-\mathrm{HP} 1 \gamma$ staining. However, staining was relatively negligible in cells that were positively marked by a short pulse of EdU, indicative of $\mathrm{S}$ phase (Figure 2G,H,I) with only $22.7 \%$ of EdU-positive cells

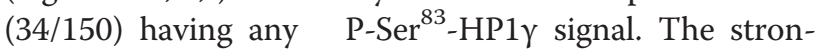
gest $\mathrm{P}-\mathrm{Ser}^{83}-\mathrm{HP} 1 \gamma$ signal was found in $88.3 \%$ of cyclin B-positive cells $(182 / 206)$, which corresponded to $G_{2}$ (Figure 2J,K,L), and the signal continued through $\mathrm{M}$ in prometaphase, metaphase and anaphase, until returning to similar levels as $G_{1}$ during telophase and cytokinesis (Figure 2M,N,O,P,Q,R). Thus, these results were congruent with our biochemical studies and confirmed that P-Ser ${ }^{83}-\mathrm{HP} 1 \gamma$ occurs as two peaks, beginning at $G_{1}$ and ending at $S$, and the second peak which begins at $G_{2}$ and continues during $M$. Interestingly, a conspicuous feature of $\mathrm{P}-\mathrm{Ser}^{83}-\mathrm{HP} 1 \gamma$ localization was its staining in cyclin B-positive cells for which the nuclear membrane has not yet disassembled (late $G_{2}$ prophase), in which the P-Ser ${ }^{83}-\mathrm{HP} 1 \gamma$ punctate pattern was stronger and present not only in euchromatin but also within centrosomes (Figure 2L). Although the cyclin Bpositive cells found in $\mathrm{M}$ demonstrated reduced $\mathrm{P}-\mathrm{Ser}$ ${ }^{83}$-HP1 $\gamma$ signal on chromosomes, a strong signal continued to localize at the centrosome region of the mitotic spindle (Figure 2M,N,O,P). In all these cases, $\mathrm{P}-\mathrm{Ser}^{83}-\mathrm{HP} 1 \gamma$ coincided with the presence of cyclin B at the centrosome. As several mitotic kinases are highly enriched at this organelle [17], these studies prompted us to identify the kinase responsible for the significant $\mathrm{P}-\mathrm{Ser}^{83}-\mathrm{HP} 1 \gamma$ event found during mitotic progression.

\section{$H P 1 \gamma$ is phosphorylated at $G_{2} / M$ by Aurora $A$}

While PKA was implicated in the first peak of P-Ser ${ }^{83}$ HP1 $\gamma$ levels that occur at $G_{1}$, the kinase that mediates the second peak of P-Ser ${ }^{83}-\mathrm{HP} 1 \gamma$ at $\mathrm{G}_{2} / \mathrm{M}$, described here, remained unknown. Interestingly, we found that the temporal pattern of $\mathrm{P}-\mathrm{Ser}^{83}-\mathrm{HP} 1 \gamma$ coincided with phosphorylation of histone $\mathrm{H} 3$ at serine $10\left(\mathrm{P}_{-} \mathrm{Ser}^{10}-\mathrm{H} 3\right.$, Figure $1 \mathrm{C}$ ). $\mathrm{P}-\mathrm{Ser}^{10}-\mathrm{H} 3$ initiates during $\mathrm{G}_{2}$ in pericentric foci and spreads along the chromosome arms, thus serving as a hallmark of mitosis [18]. Previously derived consensus sequences for Aurora kinases suggested that, similar to $\mathrm{P}-\mathrm{Ser}^{10}-\mathrm{H} 3, \mathrm{Ser}^{83}-\mathrm{HP} 1 \gamma$ might be a target of Aurora kinases [19]. Additional experiments demonstrated that the temporal pattern of $\mathrm{P}-\mathrm{Ser}^{83}-\mathrm{HP} 1 \gamma$ was similar to both Aurora A and Aurora B (Figure 1C). These results led us to hypothesize that the newly described P-Ser ${ }^{83}-\mathrm{HP} 1 \gamma$ at $\mathrm{G}_{2} / \mathrm{M}$ was achieved through the 

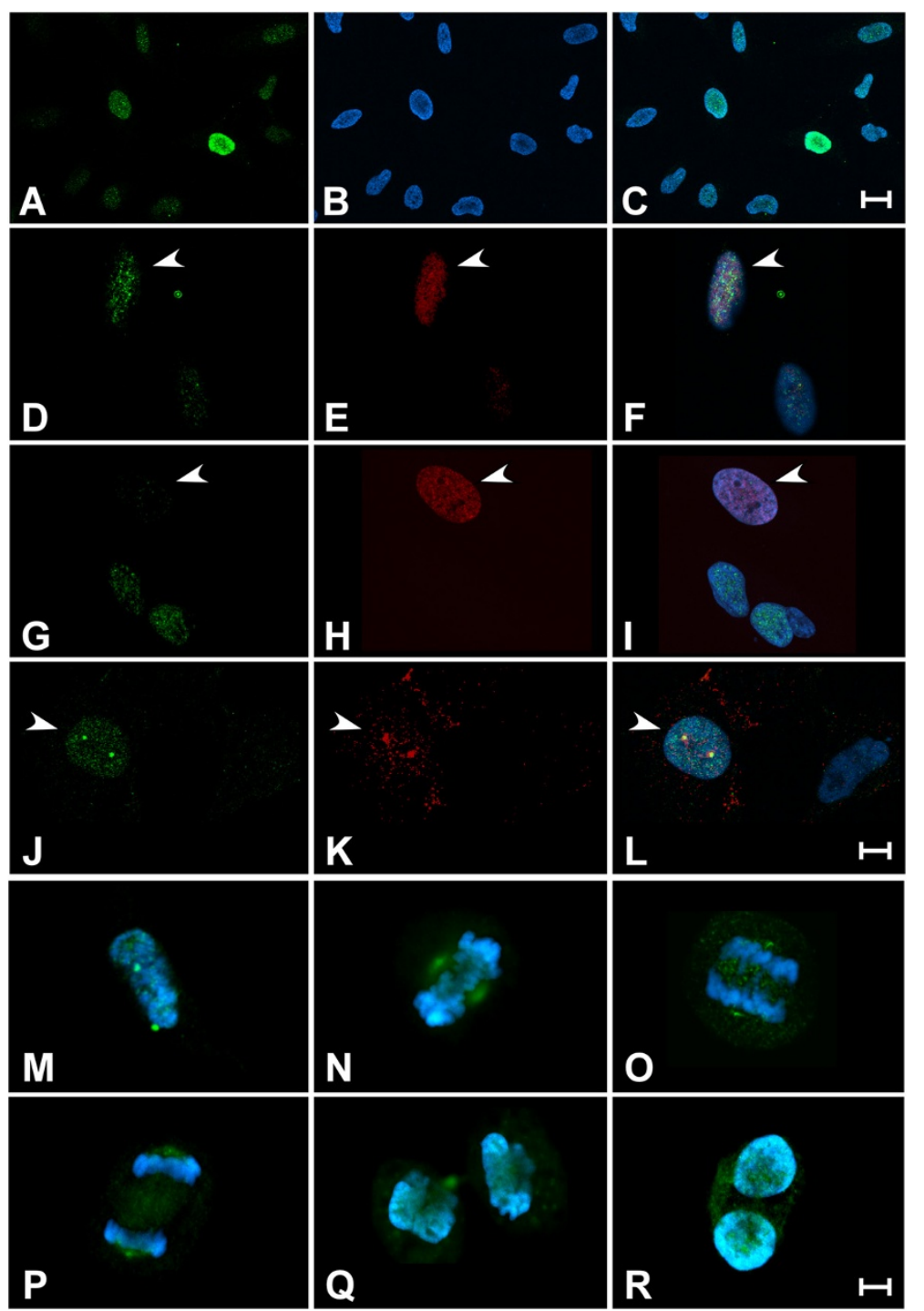

Figure 2 Biphasic P-Ser ${ }^{83}-\mathrm{HP} 1 \gamma$ is observed during cell cycle progression. (A,B,C) P-Ser ${ }^{83}-H P 1 \gamma$ levels vary during the cell cycle. Panoramic view of a growing population of HeLa cells staining with anti-P-Ser ${ }^{83}-\mathrm{HP} 1 \gamma$ (A, green) demonstrates that the signal for this protein varies in intensity in different cells. Cells were counterstained with DAPI (B, blue) to show DNA and overlay is shown in (C). Three main populations are observed according to the strength of the signal, namely strong, moderate and negligible. Scale bar represents $20 \mu M$. (D,E,F) P-Ser ${ }^{83}$-HP1Y displays punctate euchromatic localization in $G_{1}$ phase. Localization of P-Ser ${ }^{83}-H P 1 Y$ (D, green) was determined in cyclin D-positive cells (E, red), indicative of $G_{1}$ phase, as shown with arrows and in overlay (F). (G,H,I) Levels of P-Ser ${ }^{83}$-HP1Y diminish during S phase. Negligible P-Ser ${ }^{83}$-HP1Y signal (G, green) is found in the majority of cells undergoing $S$ phase (arrows), as determined by EdU positively labeled cells ( $\mathbf{H}$, red). Overlay is shown in (I). (J,K,L) P-Ser ${ }^{83}$-HP1y levels increase upon $G_{2}$ entry. Cyclin B-positive cells ( $\mathbf{K}$, red), before nuclear envelope breakdown $\left(G_{2}\right)$, not only shows the P-Ser ${ }^{83}$-HP1Y signal (J, green) as a strong punctate pattern in euchromatin, but also with separating centrosomes $(\mathbf{L}$, overlay). Scale bar represents $10 \mu \mathrm{M}$ for panels ( $\mathbf{D}$ to $\mathbf{L})$. (M,N,O,P,Q,R) P-Ser ${ }^{83}$-HP1Y levels persist through mitosis. Cyclin B-positive, prometaphase cell demonstrates an increase in P-Ser ${ }^{83}$-HP1Y in association with separating centrosomes (M). Metaphase cell shows the P-Ser ${ }^{83}$-HP1 1 remains localized to

centrosomes, which are forming the mitotic spindle $\mathbf{( N )}$. Early $\mathbf{(} \mathbf{O})$ and late $\mathbf{( P )}$ anaphase, as well as telophase $(\mathbf{Q})$ cells are shown, where the P-Ser ${ }^{83}$-HP1Y signal intensity at the centrosomes is decreased as cells prepare to complete cell division. P-Ser ${ }^{83}-\mathrm{HP} 1$ Y signal within euchromatic regions is again observed during cytokinesis (R). Scale bar represents $5 \mu \mathrm{M}$ for panels ( $\mathrm{M}$ to R). DAPI, 4',6-diamidino-2-phenylindole; EdU, 5-ethynyl-2'deoxyuridine; P-Ser ${ }^{83}$-HP1 1 , phosphorylation of HP1 $\gamma$ at serine 83.

activity of an Aurora kinase. Thus, we first performed immunofluorescence experiments to determine whether P$\mathrm{Ser}^{83}$-HP1Y co-localized with any of these kinases at $\mathrm{G}_{2} /$ $\mathrm{M}$. Indeed, we found that $\mathrm{P}-\mathrm{Ser}^{83}-\mathrm{HP} 1 \gamma$ localized to areas rich in Aurora A (Figure $3 \mathrm{~A}, \mathrm{~B}, \mathrm{C}$ ), but not Aurora B
(Figure 3D,E,F). P-Ser ${ }^{83}$-HP1 1 was also confirmed to be present at the Aurora A-rich area of the spindle poles through colocalization with $\gamma$-tubulin (Figure 3G,H,I) and $\alpha$-tubulin (Figure 3J,K,L). More importantly, we found that critical regulators of $\mathrm{G}_{2} / \mathrm{M}$ progression, which are 

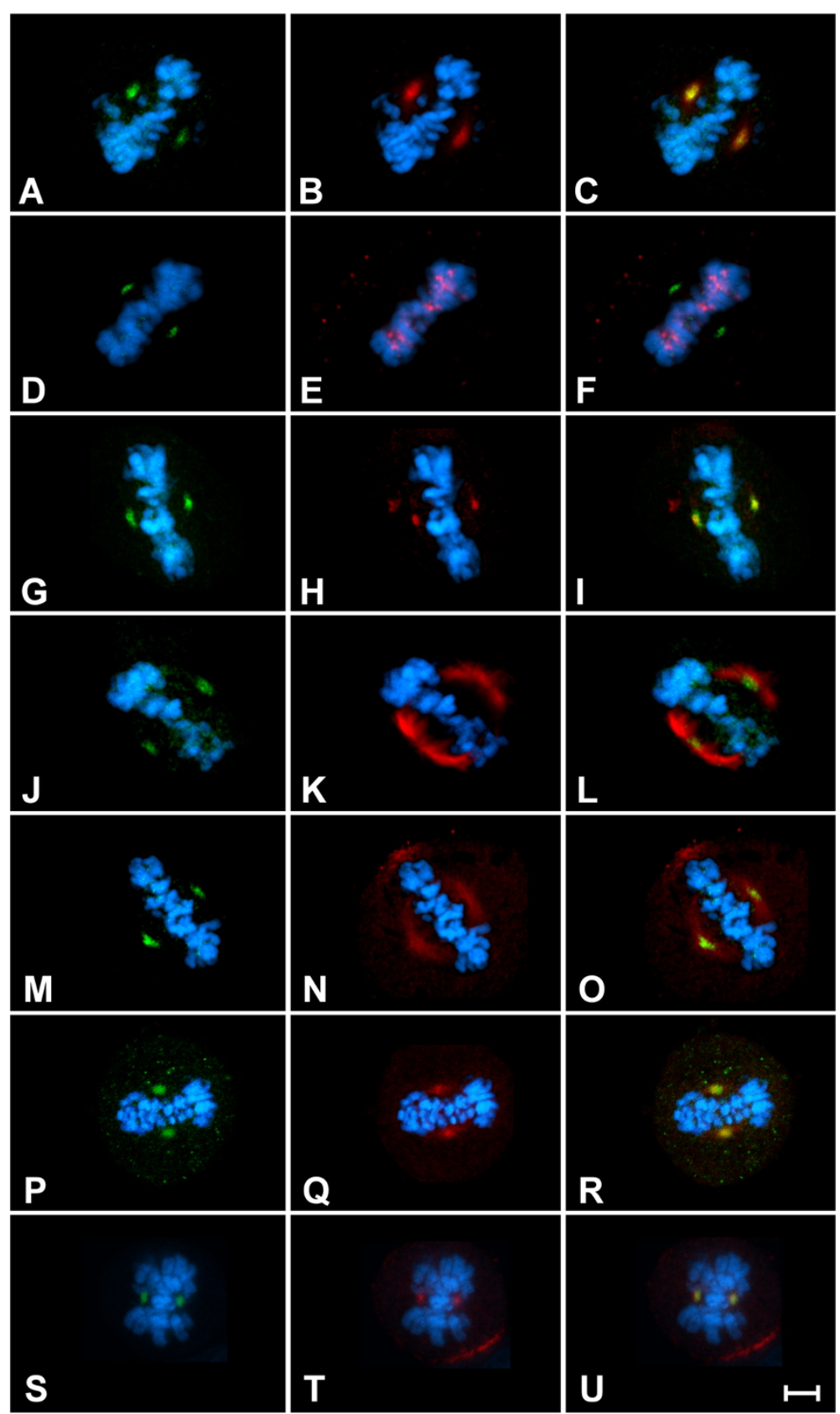

Figure 3 P-Ser ${ }^{83}-\mathrm{HP} 1 \gamma$ colocalizes with Aurora A at the mitotic spindle. Representative images are shown for localization in mitotic HeLa cells. $(\mathbf{A}, \mathbf{B}, \mathbf{C})$ Colocalization of P-Ser ${ }^{83}-\mathrm{HP} 1 \mathrm{Y}(\mathbf{A}$, green) is shown with Aurora A (B, red) at the spindle poles. The overlay is shown in $(\mathbf{C})$. (D,E,F) Cells in metaphase were also stained for P-Ser ${ }^{83}-H_{1} 1 \gamma(\mathbf{D}$, green) and Aurora B (E, red), which demonstrates that there is no colocalization of these two proteins as observed in the overlay $(\mathbf{F}) .(\mathbf{G}, \mathbf{H}, \mathbf{I}, \mathbf{J}, \mathbf{K}, \mathbf{L}) \mathbf{P}-\mathrm{Ser}^{83}-\mathrm{HP} 1 \mathbf{Y}(\mathbf{G}, \mathbf{J}$, green) was confirmed to be present at the spindle poles through co-staining with $\gamma$-tubulin ( $\mathbf{H}$, red) as well as a-tubulin (K, red) as shown in the overlays (I, $\mathbf{L})$. (M, $\mathbf{N}, \mathbf{O}, \mathbf{P}, \mathbf{Q}, \mathbf{R}, \mathbf{S}, \mathbf{T}, \mathbf{U})$ In addition, CDK1 ( $\mathbf{N}$, red), cyclin B1 ( $\mathbf{Q}$, red) and cyclin B2 ( $\mathbf{T}$, red) were each shown to co-localize with P-Ser ${ }^{83}-H P 1 \gamma(\mathbf{M}, \mathbf{P}, \mathbf{S}$, green) as shown by overlays $(\mathbf{O}, \mathbf{R}, \mathbf{U})$. Cells were counterstained with DAPI (blue) to show DNA. Scale bar represents $5 \mu \mathrm{M}$. CDK1, cyclin-dependent kinase 1; DAPI, 4',6-diamidino-2phenylindole; P-Ser ${ }^{83}-\mathrm{HP} 1 \gamma$, phosphorylation of HP1 1 at serine 83.

also targets of Aurora A, namely cyclin B1, cyclin B2 and their partner kinase, CDK1, also colocalized with P-Ser ${ }^{83}$ HP1 $\gamma$ (Figure 3M,N,O,P,Q,U). Together, these results demonstrated that mitotic phosphorylation confers a distinct localization of this $\mathrm{HP} 1 \gamma$ subpopulation to the spindle poles that is marked by the $\mathrm{G}_{2} / \mathrm{M}$ Aurora A-cyclin $\mathrm{B}-\mathrm{CDK} 1$ pathway, supporting the idea that this kinase may be the enzyme involved in $\mathrm{P}-\mathrm{Ser}^{83}-\mathrm{HP} 1 \gamma$ at $\mathrm{G}_{2} / \mathrm{M}$.

To mechanistically test this hypothesis, we initially incubated glutathione S-transferase (GST) fusion wild type 
and nonphosphorylatable mutant $\mathrm{HP} 1 \gamma$ proteins with each Aurora kinase, Aurora A or Aurora B, followed by western blot using the phospho-specific $\mathrm{P}-\mathrm{Ser}^{83}-\mathrm{HP} 1 \gamma$ antibody. These in vitro kinase assays demonstrated that the wild type $\mathrm{HP} 1 \gamma$, but not the dominant negative, nonphosphorylatable S83A-HP1 $\gamma$ mutant [8], could be phosphorylated in vitro by both Aurora A and Aurora B (Figure 4A). To determine whether Aurora kinases also phosphorylate HP1y in vivo, we performed western blots of siRNA-treated HeLa cells against Aurora A and B, separately (Figure 4B). We found that Aurora A siRNA can inhibit the $\mathrm{P}-\mathrm{Ser}^{83}-\mathrm{HP} 1 \gamma$ in vivo, whereas Aurora B siRNA demonstrated only a slight reduction in levels of P-Ser ${ }^{83}-\mathrm{HP} 1 \gamma$ (56\% of control levels). Of note, Aurora A kinase depletion by siRNA also leads to arrest of cells at $\mathrm{G}_{2} / \mathrm{M}$ [20], thus eliminating the influence of the $G_{1}$

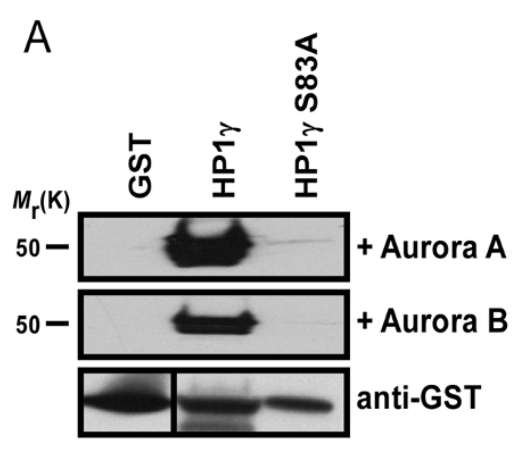

B
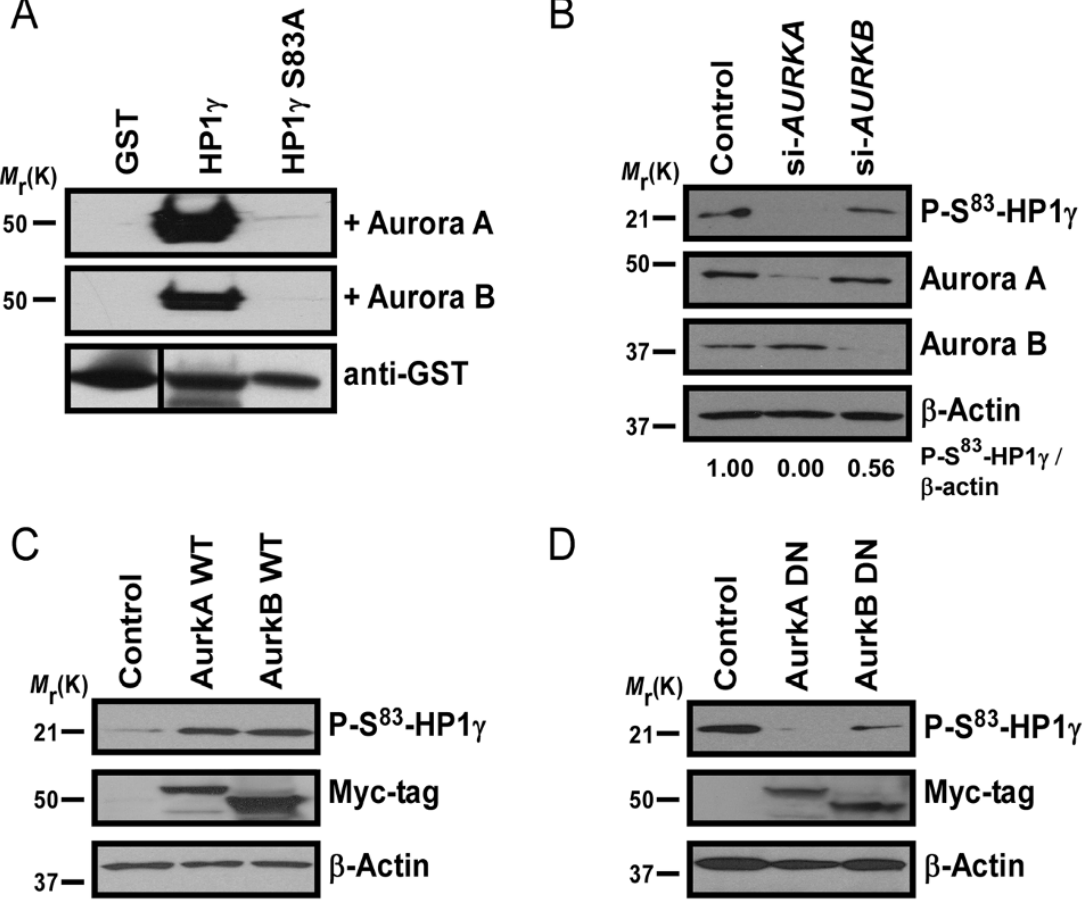

E

F

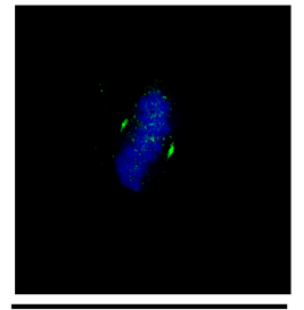

Control

G

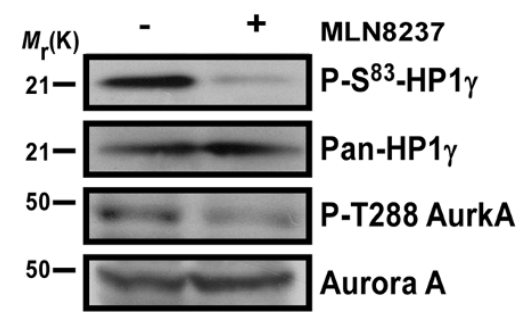

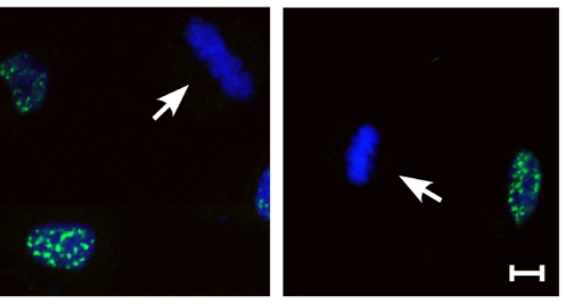

Aurora A DN

$\mathrm{H}$

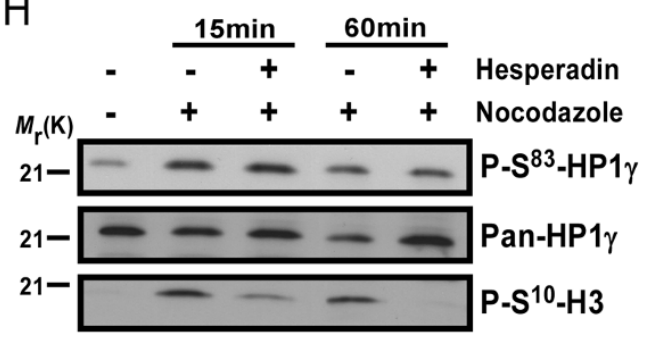


(See figure on previous page.)

Figure 4 Aurora A phosphorylates Ser ${ }^{83}-\mathrm{HP}_{1} \boldsymbol{\gamma}$ in $\mathrm{G}_{2} / \mathbf{M}$. (A) Aurora kinases phosphorylate $\mathrm{Ser}^{83}$ in vitro. In vitro kinase assays were performed on GST fusion proteins, which demonstrate that wild type, not S83A-HP1Y mutant, is phosphorylated by Aurora kinases. (B) Aurora A siRNA reduces P-Ser ${ }^{83}-\mathrm{HP} 1 \gamma$. Aurora A siRNA significantly reduced P-Ser ${ }^{83}-\mathrm{HP} 1 \gamma$, whereas Aurora B siRNA only slightly reduced P-Ser ${ }^{83}-\mathrm{HP} 1 \gamma$ (top). Aurora $\mathrm{A}(A \cup R K A)$ and Aurora B (AURKB) were effectively knocked-down (middle panels). Relative intensities were calculated as P-Ser ${ }^{83}$-HP1 $\gamma / \beta$-actin ratios. (C) Wild type Aurora kinases increase P-Ser ${ }^{83}-\mathrm{HP} 1 \gamma$. CHO cells, with low basal P-Ser ${ }^{83}-\mathrm{HP} 1 \gamma$, demonstrated increased P-Ser ${ }^{83}-\mathrm{HP} 1 \gamma$ (top) upon transfection of Aurora kinases (Myc-tag; middle). (D) Aurora A-dominant negative (DN) reduces P-Ser ${ }^{83}$-HP1Y. P-Ser ${ }^{83}$-HP1Y (top) was significantly reduced with Aurora A-DN in BxPC3, epithelial cells with high basal P-Ser ${ }^{83}-\mathrm{HP} 1$. A Arora B-DN also reduced P-Ser ${ }^{83}$-HP1Y, although still detected. Aurora-DN levels are shown by Myc-tag. $\beta$-actin serves as loading control (B, C, D; bottom). (E,F) Aurora A-DN abolishes mitotic P-Ser ${ }^{83}$-HP1Y. Representative images of overlays with DAPI counterstain are shown for P-Ser ${ }^{83}-\mathrm{HP} 1$ ( (green) with control (E) or Aurora A-DN (F). Typical P-Ser ${ }^{83}$ HP1y localization was still observed in interphase with Aurora A-DN, but disrupted in metaphase (arrows). Scale bar represents $10 \mu \mathrm{M}$. (G,H). Pharmacological inhibition of Aurora A, but not Aurora B, inhibits P-Ser ${ }^{83}$-HP1Y. Aurora A inhibition with MLN8237 was confirmed by loss of activated P-Thr ${ }^{288}$ relative to total Aurora A (G, lower panels). P-Ser ${ }^{83}-\mathrm{HP} 1 \mathrm{Y}$ was significantly reduced with MLN8237, without affecting pan-HP1Y (G, upper panels). Conversely, Aurora B inhibition by hesperidin did not reduce P-Ser ${ }^{83}-H P 1 Y(H$, top). Aurora B inhibition was confirmed by P-Ser ${ }^{10}$-H3, a well-known Aurora B target (H, bottom). CHO, Chinese hamster ovary; DAPI, 4',6-diamidino-2-phenylindole; DN, dominant negative; GST, glutathione S-transferase; P-Ser ${ }^{10}-\mathrm{H} 3$, phosphorylation of histone $\mathrm{H3}$ at serine 10; P-Ser ${ }^{83}-\mathrm{HP} 1$, phosphorylation of HP1 $\gamma$ at serine 83; P-Thr ${ }^{288}$, phosphorylation of Aurora A at threonine 288; $\mathrm{Ser}^{83}$, serine 83.

phosphorylation in these experiments. To further investigate the participation of Auroras in this event, Chinese hamster ovary $(\mathrm{CHO})$ cells, which have relatively low basal levels of P-Ser ${ }^{83}-\mathrm{HP} 1 \gamma$, were transfected with either wild type Aurora A or Aurora B (Figure 4C). As a result, levels of $\mathrm{P}-\mathrm{Ser}^{83}-\mathrm{HP} 1 \gamma$ were higher in the Aurora-transfected cells than control. This occurred with both Aurora A and Aurora B transfection, as expected due to their effects on cell cycle progression. In contrast, transfection of epithelial cells, BxPC3, which have high basal levels of P-Ser ${ }^{83}$ $\mathrm{HP} 1 \gamma$, with the dominant negative form of Aurora A $[21,22]$ resulted in reduced levels of $\mathrm{P}-\mathrm{Ser}^{83}-\mathrm{HP} 1 \gamma$ (Figure $4 \mathrm{D})$. Similar to the siRNA experiments, dominant negative Aurora B $[21,23]$ had less effect on P-Ser ${ }^{83}-\mathrm{HP} 1 \gamma$ levels than Aurora A. Therefore, we utilized the dominant negative Aurora A in HeLa cells to confirm this phenomenon by immunofluorescence. Compared to control cells (Figure 4E), transfections with dominant negative Aurora A (Figure 4F) abolished the localization of the P$\mathrm{Ser}^{83}-\mathrm{HP} 1 \gamma$ in cell compartments rich in this kinase (arrow). In contrast, transfection with dominant negative Aurora A did not affect P-Ser ${ }^{83}-\mathrm{HP} 1 \gamma$ levels or localization in interphase cells (Figure 4F). Furthermore, we utilized the Aurora A- and Aurora B-specific pharmacological inhibitors, MLN8237 and hesperidin, respectively, to determine the participation of each kinase in P-Ser ${ }^{83}-\mathrm{HP} 1 \gamma$. We found that specific inhibition of Aurora A with 300 nM MLN8237, which was confirmed by loss of activated phosphorylation of Aurora A at threonine 288 $\left(\mathrm{P}-\mathrm{Thr}^{288}\right)$ [24], diminished P-Ser ${ }^{83}-\mathrm{HP} 1 \gamma$ levels without affecting total HP1 $\gamma$ protein levels (Figure 4G). However, treatment with hesperidin $(200 \mathrm{nM})$ for specific inhibition of Aurora B did not reduce P-Ser ${ }^{83}$-HP1 1 levels, while still inhibiting the Aurora B target P-Ser ${ }^{10}-\mathrm{H} 3$ (Figure 4H, and personal communication with $\mathrm{H}$ Dormann and CD Allis). Combined, these results demonstrate that Aurora A kinase is primarily responsible for the localization and increased level of $\mathrm{P}-\mathrm{Ser}^{83}-\mathrm{HP} 1 \gamma$ observed in $\mathrm{G}_{2} / \mathrm{M}$.
Together with the biochemical experiments described above, these data implicate, for the first time, Aurora kinase in the cell cycle-regulated P-Ser ${ }^{83}-\mathrm{HP} 1 \gamma$. This observation also represents the first evidence describing mammalian HP1 at the spindle poles, a localization that is preferred by a large amount of proteins involved in the regulation of cell cycle transitions.

\section{P-Ser ${ }^{83}-\mathrm{HP} 1 \mathrm{\gamma}$ is required for normal mitotic function}

Functionally, HP1 $\gamma$ has been previously shown to play a role in cell cycle progression [10-13], although how this protein is regulated to modulate this function remains unknown. Inhibition of Aurora A leads to mitotic spindle defects and misaligned chromosomes [25,26]. Thus, as phosphorylation of $\mathrm{HP} 1 \gamma$ is downstream of this pathway during mitosis, we investigated whether disrupting the function of this protein also coincides with this effect. For this purpose, we performed stable lentiviral-

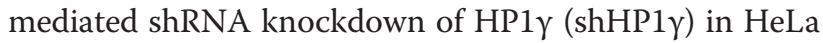
cells. HP1 $1 \gamma$ knockdown was confirmed by western blot with approximately $90 \%$ reduction in protein levels (Figure 5A). These cells also displayed a significant decrease in $\mathrm{P}-\mathrm{Ser}^{83}-\mathrm{HP} 1 \gamma$ staining by immunofluorescence (Figure $5 \mathrm{~B}$ ), demonstrating that localization of $\mathrm{P}-\mathrm{Ser}^{83}$ $\mathrm{HP} 1 \gamma$ to the mitotic spindle pole was unambiguous. We found that $25.5 \%$ of shHP1 $\gamma$ cells in mitosis displayed abnormalities ( $n=200$, Figure $5 C$ ), including multipolar spindles, centrosome disruption or lagging, unorganized chromosomes (Figure 5D). shRNA control cells (shCTRL) displayed abnormalities in only $1 \%(\mathrm{n}=200)$. However, in spite of this informative outcome, since HP1 1 knockdown depleted all forms of the protein, the contribution of $\mathrm{Ser}^{83}$ phosphorylation to this effect could not be assessed by this experimental manipulation. Thus, to better determine the role that phosphorylation of $\mathrm{Ser}^{83}$ plays in this function, we sought to rescue the knockdown phenotype with wild type and $\operatorname{Ser}^{83}$ mutant $\mathrm{HP} 1 \gamma$. Transduction with empty vector (EV) control did 


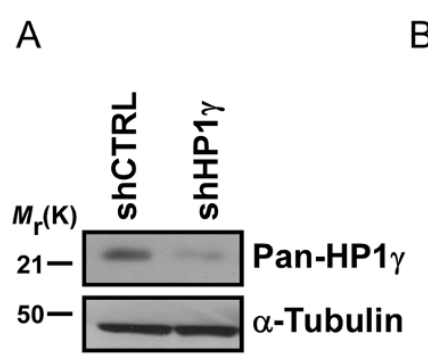

B
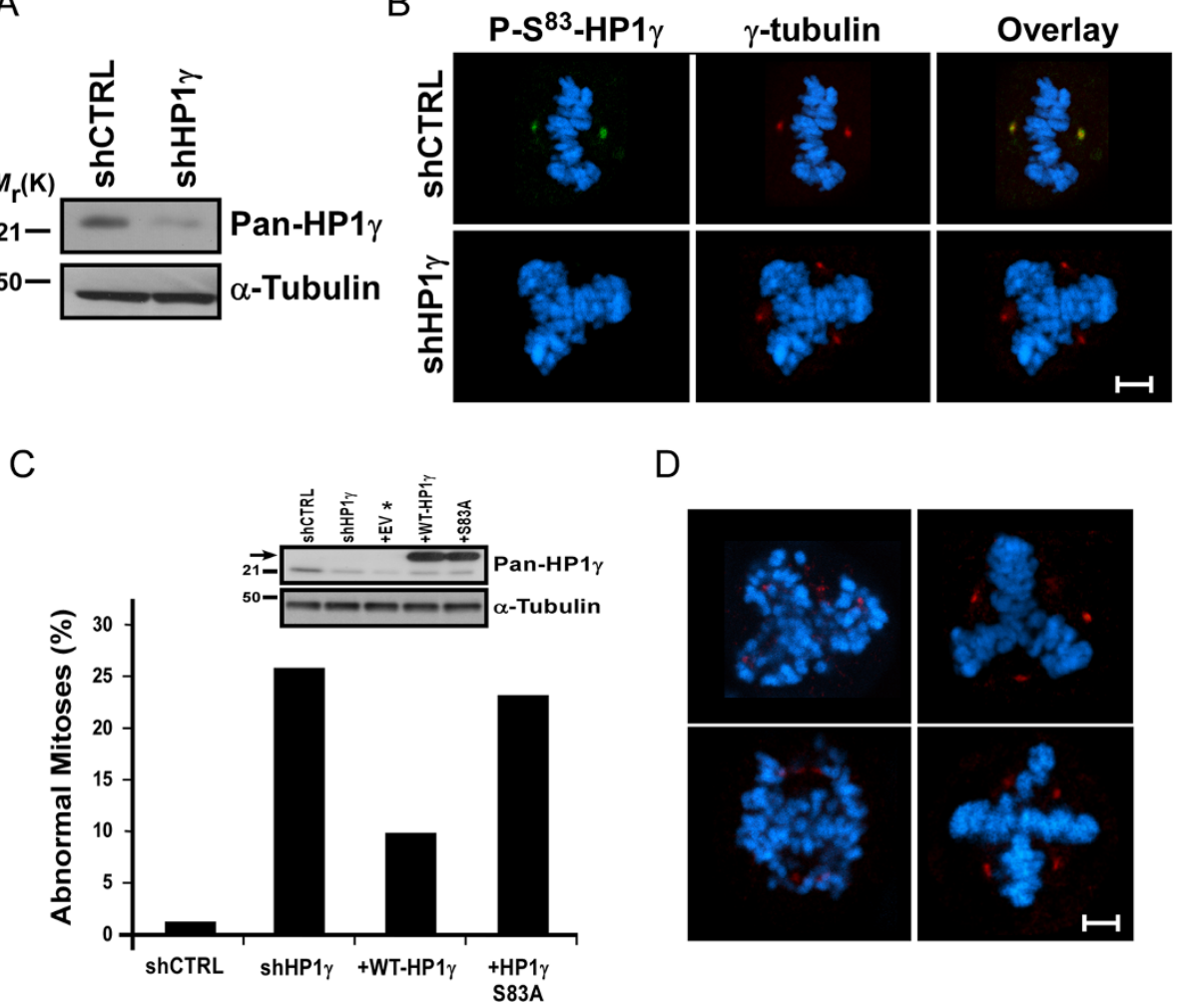

D

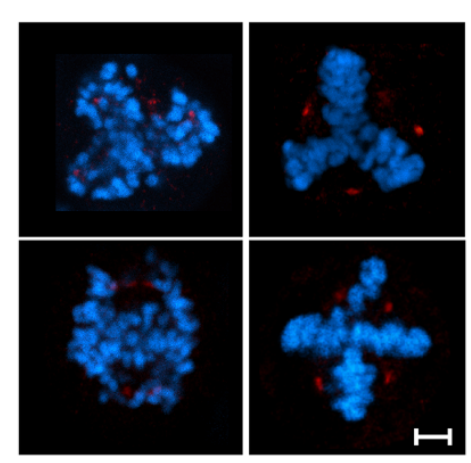

Figure 5 P-Ser ${ }^{83}-\mathrm{HP} 1 \boldsymbol{\gamma}$ is necessary for proper mitotic function. (A) Stable knockdown of HP1Y in HeLa cells. Western blot of HP1 $y$ levels (top) is shown from HeLa cell lysates to confirm stable lentiviral-mediated shHP1Y compared to shCTRL. a-tubulin serves as a loading control (bottom). (B) HP1 1 knockdown eliminates P-Ser ${ }^{83}$-HP1 1 at the spindle poles. Representative images are shown for immunofluorescence on

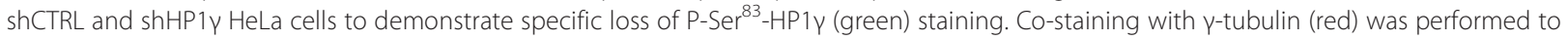
establish the localization of the spindle poles. Cells were counterstained with DAPI and the overlay is shown. Scale bar represents $5 \mu$ M. (C) Mitotic aberrations caused by HP1Y knockdown are rescued by wild type, but not S83A-HP1 1 mutant. Mitotic aberrations were quantified for shCTRL and shHP1y cells. In order to determine if $\mathrm{Ser}^{83}$ phosphorylation plays a role in this function, shHP1Y cells were infected with adenovirus carrying wild type or S83A-HP1 $\gamma$ mutant. While reintroduction of wild type HP1 $y$ was able to significantly rescue this effect, S83A-HP1Y mutant was not, implicating Aurora A-mediated phosphorylation in this phenomenon. For each condition, 200 mitotic cells were analyzed. Western blot is shown of endogenous HP1Y levels (inlay, top) as well as transduced His-tagged wild type and S83A-HP1Y mutant proteins (arrow). a-tubulin serves as a loading control (inlay, bottom). *Transduction with EV control did not change the number of abnormalities observed with shHP1Y. (D) Mitotic aberrations observed in stable shHP1Y cells include multipolar spindles, centrosome disruption and lagging, unorganized chromosomes. Representative images are shown for the types of observed mitotic aberrations. $\gamma$-tubulin (red) marks spindle poles with DAPI counterstain to show condensed mitotic chromosomes. Scale bar represents $5 \mu \mathrm{M}$. DAPI, 4',6-diamidino-2-phenylindole; EV, empty vector; P-Ser ${ }^{83}-\mathrm{HP}_{1} \gamma$, phosphorylation of HP1 1 at serine 83; $\mathrm{Ser}^{83}$, serine 83; shCTRL, shRNA control; shHP1 $\gamma$, shRNA knockdown of HP1 $\gamma$; shRNA, short hairpin RNA.

not change the number of abnormalities observed with

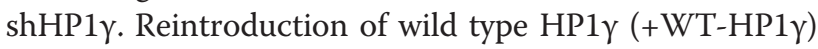
rescued, to a significant extent, the abnormal mitotic effects seen with knockdown of this protein (10\% abnormal, $\mathrm{n}=200)$. Notably, an alanine substitution, which rendered $\mathrm{HP} 1 \gamma$ unable to undergo phosphorylation at $\operatorname{Ser}^{83}$ (+S83A), was unable to rescue the knockdown phenotype $(23 \%$ abnormal, $\mathrm{n}=200)$. This data indicates that first, normal $\mathrm{HP} 1 \gamma$ levels are necessary for proper mitotic functions and second, HP1 $1 \gamma$ must be amenable to Aurora A-mediated $\operatorname{Ser}^{83}$ phosphorylation to achieve these effects.
P-Ser ${ }^{83}$-HP1Y status affects cell proliferation and mitotic gene expression networks

Normal mitotic cell division is a prerequisite for proliferative homeostasis and proper cell cycle progression [27]. Thus, based on our results demonstrating the role of $\mathrm{P}$ $\mathrm{Ser}^{83}-\mathrm{HP} 1 \gamma$ in mitosis, we examined the resultant effects of $\mathrm{P}-\mathrm{Ser}^{83}-\mathrm{HP} 1 \gamma$ on cell proliferation by analyzing cells

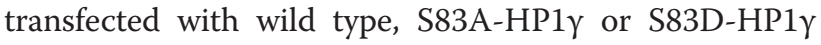
mutant via EdU incorporation. We found that wild type $\mathrm{HP} 1 \gamma$ had a slight increase in EdU incorporation compared to EV control $(103.9 \% \pm 2.6 \%$ of EV control, Figure 6A). However, nonphosphorylatable S83A-HP1 $\gamma$ 
mutant decreased the levels of EdU $(94.2 \% \pm 1.6 \%$ of EV control, $P<0.05$, Figure 6A). Notably, an aspartic acid substitution (S83D), designed to mimic $\operatorname{Ser}^{83}$ phosphorylation, had a significant increase in levels of EdU incorporation over control cells $(111.2 \% \pm 2.6 \%$ of $\mathrm{EV}$ control, $P<0.05$, Figure $6 \mathrm{~A}$ ). Thus, these results support the idea that phosphorylation of $\mathrm{Ser}^{83}$ is necessary for the regulation of cell cycle progression by $\mathrm{HP} 1 \gamma$.

We next investigated whether the changes observed in EdU incorporation by both phosphomimetic and nonphosphorylatable $\mathrm{Ser}^{83}-\mathrm{HP} 1 \gamma$ mutants were accompanied by changes in other biochemical surrogates for cell cycle progression, such as known mitotic gene networks. For this purpose, we performed a genome-wide query using Affymetrix (Santa Clara, CA, USA) profiles as transcriptional readouts of their effects. Hierarchical clustering of targets significantly altered by $\mathrm{HP} 1 \gamma$ (526 targets),

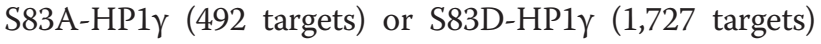
overexpression demonstrated that gene networks modulated by $\mathrm{HP} 1 \gamma$ experienced deregulation in the presence of

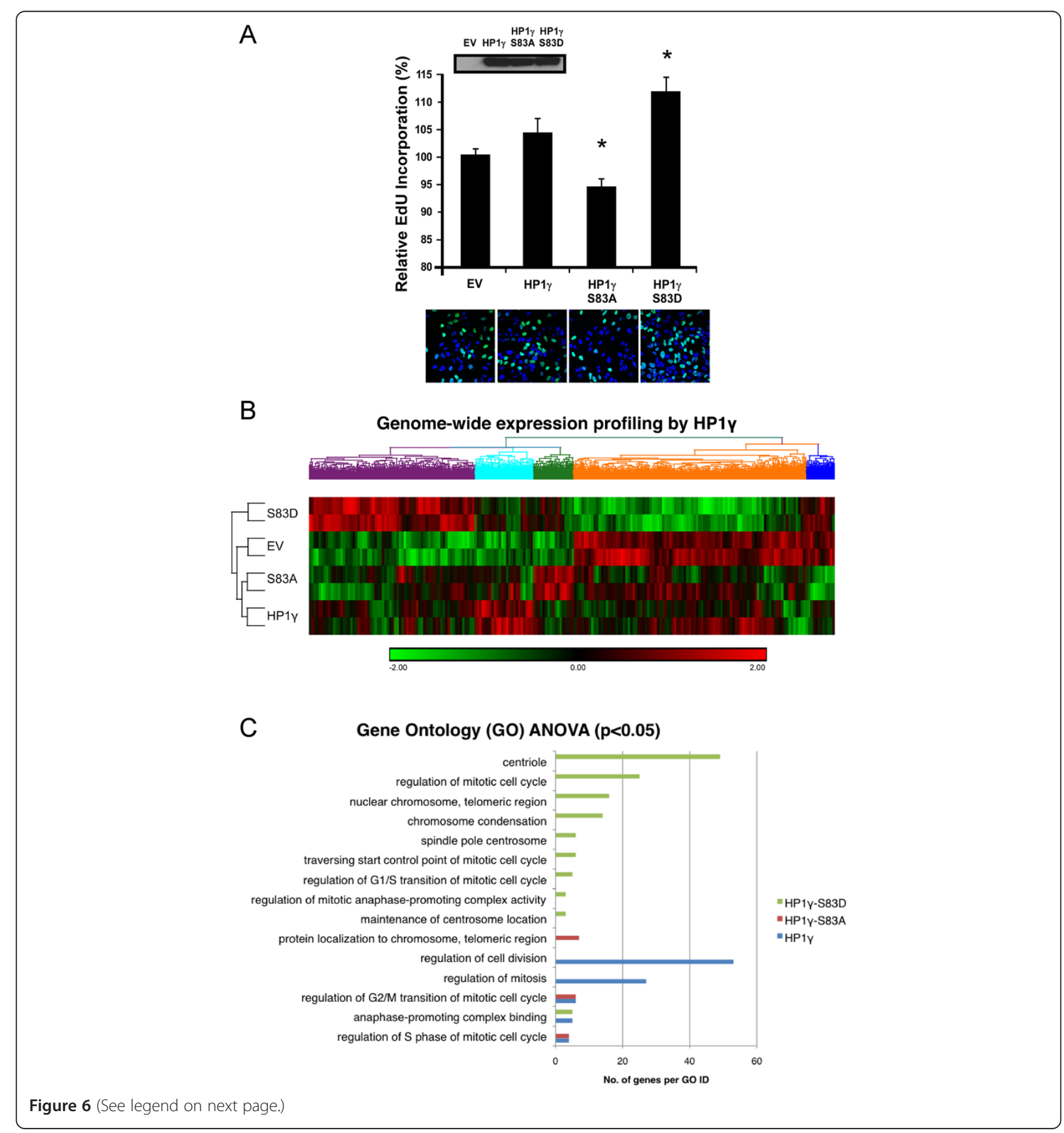




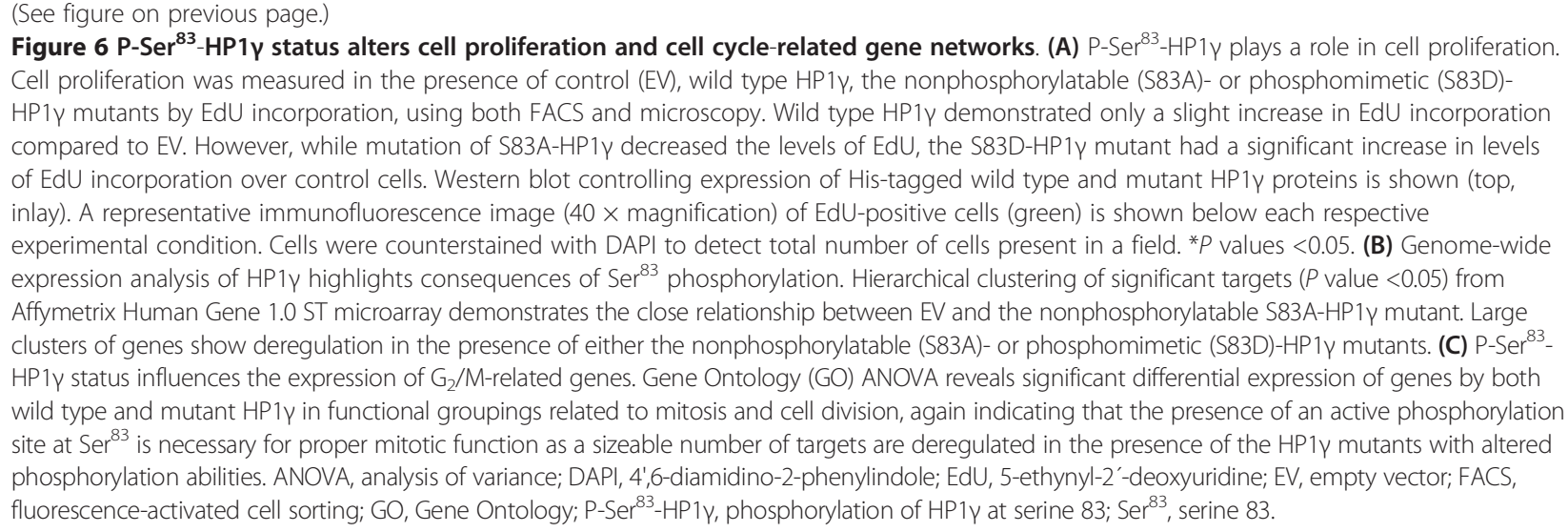

the $\operatorname{Ser}^{83}$ mutation, indicating dependence of these processes on regulation of $\operatorname{Ser}^{83}$ phosphorylation (Figure 6B). Based on Euclidean distance calculation and the resulting dendrogram, both control and nonphosphorylatable S83A-HP1 $\gamma$ mutant samples were statistically the most similar (Figure 6B). The fact that the EV and the S83AHP1 $\gamma$ mutant possessed the closest relationship suggested that the latter worked predominantly as either an inactive or dominant negative mutant. However, the phosphomimetic S83D-HP1 $\gamma$ mutant, for the most part, reversed the effect of the S83A-HP1 $\gamma$ mutant, suggesting that it likely worked in a constitutively active manner thereby mimicking Aurora Amediated $\mathrm{Ser}^{83}$ phosphorylation. Pathway-specific RT-PCR was used to validate a subset of significant targets (Additional file 2: Table S1). These experiments revealed that $\mathrm{HP} 1 \gamma$ and its phosphorylated form have the ability to change the levels of transcripts related to mitosis.

Gene Ontology (GO) ANOVA analysis was utilized to probe for differentially expressed functional groupings of genes (Figure 6C). Overall, HP1 1 overexpression resulted in significant enrichment of targets related to regulation of cellular proliferation, cell division, and mitosis $(P<0.05)$. S83A-HP1 $\gamma$ mutant overexpression yielded differential expression in targets related to protein localization to the chromosome, regulation of the $\mathrm{S}$ phase of the mitotic cell cycle and regulation of the $\mathrm{G}_{2} / \mathrm{M}$ transition of mitotic cell cycle. S83D-HP1 $\gamma$ mutant overexpression showed significant alteration in genes related to the regulation of the mitotic cell cycle, regulation of the $G_{2} / M$ anaphasepromoting complex, maintenance of centrosome location and spindle pole structure, among others. Consequently, from these data, we conclude that disruption of phosphorylation status of HP1 $\gamma$ has diverse effects on multiple aspects of the mitotic cell cycle, which is congruent with its cell cycle-associated phosphorylation pattern (Figures 1 and 2) indicating a pervasive role of the regulation of $\mathrm{HP} 1 \gamma$ in cell division.

Interestingly, previous studies have shown that depletion of HP1 $\gamma$ in primordial germ cells reduces their number as a result of impaired cell cycle progression [13]. Comparison of our expression dataset with a published dataset in primordial germ cells revealed that the expression of the nonphosphorylatable S83A-HP1 $\gamma$ mutant displayed a highly similar pattern as HP1 $\gamma$ depletion, including targets related to cell cycle, proliferation and

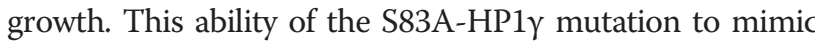
conditions of absolute HP1 $\gamma$ depletion at the level of gene expression networks, combined with the inability of the S83A-HP1 $\gamma$ mutant to rescue the mitotic defects observed with $\mathrm{HP} 1 \gamma$ knockdown, indicates that posttranslational modification of this residue is needed for proper progression through mitosis. Furthermore, it may be concluded from our genome-wide analysis that $\mathrm{HP} 1 \gamma$ participates in the regulation of processes, which support proper cell division and proliferation through phosphorylation-dependent and phosphorylation-independent mechanisms.

\section{Discussion}

Based on the current study, our demonstration that $\mathrm{HP} 1 \gamma$, a well-known epigenetic regulator, undergoes robust phosphorylation at $\operatorname{Ser}^{83}$ in $\mathrm{G}_{2} / \mathrm{M}$ has significant biological relevance and deserves careful consideration. Previous studies demonstrating that HP1 proteins are ejected from chromosomes during mitosis $[28,29]$ led to the assumption that this protein is not involved in the regulation of this process, even though it is highly express in rapidly dividing cancer cells [10]. In this regard, the current study reveals that, during $\mathrm{G}_{2} / \mathrm{M}$, an extrachromosomal subpopulation of HP1 $\gamma$, P-Ser ${ }^{83}$-HP1 $\gamma$, localizes with $\gamma$-tubulin, Aurora A kinase and other mitotic targets, including cyclin B1, cyclin B2 and CDK1, at the spindle poles. Thus, this data demonstrates for the first time that, in spite of its ejection from chromosomes, HP1 $\gamma$ does not disappear during mitosis, but rather relocates to organelles, known for enrichment in cell cycle regulators, where it undergoes $G_{2} / M$-specific phosphorylation at $\operatorname{Ser}^{83}$ by Aurora A. In addition, the colocalization and coupling of Aurora A to $\mathrm{HP} 1 \gamma$ in cell 
cycle regulation is reconstituted in time and space in each cell cycle.

Examination of the effect of the related kinase, Aurora $B$, demonstrates that this enzyme can phosphorylate the $\mathrm{Ser}^{83}$ site in vitro. However, siRNA and dominant negative experiments demonstrate that Aurora B was not as robust as Aurora A on modulating levels of $\mathrm{P}-\mathrm{Ser}^{83}$ HP1 $\gamma$ in cells. Treatment of cells with the Aurora B inhibitor, hesperidin, does not impair $\mathrm{P}-\mathrm{Ser}^{83}-\mathrm{HP} 1 \gamma$ and, more importantly, Aurora B does not localize with P-Ser ${ }^{83}$-HP1Y in mitotic cells. These results reveal a significant level of specificity for these kinases in the phosphorylation of HP1 proteins.

We found that HP1y, though ejected from chromosomes by the previously described Aurora-mediated P$\mathrm{Ser}^{10}-\mathrm{H} 3[28,29]$, remains tightly associated to a mitotic organelle which is rich in cell cycle regulators. This reveals the existence of coupled mechanisms of ejection and relocalization of $\mathrm{HP} 1 \gamma$, which ultimately has significant consequences for the regulation of cell division. Both steps involved in this process, $\mathrm{H} 3$ and $\mathrm{HP} 1 \gamma$ phosphorylation, are mediated by Aurora kinases. Thus, it is most likely that one function of Auroras has evolved, in part, to secure that epigenetic regulators are turned on and off during cell division in a highly synchronized manner, to achieve the proper transfer of geneticepigenetic material through generations. Interestingly, although HP1 proteins themselves have not been previously observed at the centrosome/spindle pole, several HP1-interacting proteins are known to reside in this cell compartment. For example, a subpopulation of origin recognition complex 2 (Orc2) protein has been localized to centrosomes [30]. However, contrary to the Aurora A-cyclin B-CDK1 pathway, which links the phosphorylation of HP1 $\gamma$ at the spindle during $G_{2} / M$ transition, Orc2 associates with HP1 only in the population that is tightly bound to heterochromatin in $G_{1}$ and early $S$ phase. In addition, immunoprecipitation of Orc2 shows specific interaction with $\mathrm{HP} 1 \alpha$ and $\mathrm{HP} 1 \beta$, but not $\mathrm{HP} 1 \gamma$ [30], the HP1 protein studied here. Since posttranslational modifications of HP1 were not considered in the Orc2 experiments, it remains possible that subpopulations of distinct posttranslationally modified HP1 proteins, such as $\mathrm{P}-\mathrm{Ser}^{83}-\mathrm{HP} 1 \gamma$, which cannot be detected with pan-HP1 antibodies, also interact with Orc2. It is not likely, however, that Orc2 is responsible for recruitment of HP1y to this cell compartment, given that Orc2 is localized there throughout the entire cell cycle [30]. Nevertheless, our results demonstrate a high degree of selectivity for $\mathrm{HP} 1 \gamma$ to work with certain regulatory enzymes (kinases) to maintain mitotic functions.

Previous studies have shown that disruption of G9a, one of the histone methyltransferases responsible for the histone mark recognized and bound by HP1, H3 lysine
9, results in chromosome instability along with centrosome abnormalities [31]. In addition to creating the mark to which HP1 binds, G9a localizes with HP1 $\alpha$ and HP1 $\gamma$, which is dependent upon its own automethylation [32], and HP1Y has been shown to specifically form complexes with G9a in the context of the E2F-6 gene silencing complex [33]. Interestingly, in meiosis cell division during gamete production, HP1 $\gamma$ and G9a are proposed to form an axis that is responsible for retaining centromeric regions of unpaired homologous chromosomes in close alignment, and facilitating progression of their pairing in early meiotic prophase [12]. In fact, HP1 $\gamma$-deficient mouse spermatocytes undergo meiotic catastrophe [12]. An important observation of our studies is that siRNA-mediated knockdown of HP1 $\gamma$ leads to a decrease of P-Ser ${ }^{83}-\mathrm{HP} 1 \gamma$ accompanied by mitotic aberrations. While reintroduction of wild type HP1Y rescues, to a significant extent, these abnormal mitotic effects, the nonphosphorylatable S83A-HP1 $\gamma$ mutant is unable to rescue this consequence of $\mathrm{HP} 1 \gamma$ knockdown, highlighting the importance of $\mathrm{Ser}^{83}$ modification for this function. Moreover, the S83D-HP1 $\gamma$ mutant that mimics Aurora A phosphorylation facilitates cell proliferation, whereas the nonphosphorylatable S83A-HP1Y mutant inhibits this process. Therefore, it is tempting to speculate whether modifications of HP1 influence interactions with G9a and whether these proteins function together in regulating proper cell division. Indeed, additional studies using model organisms support that the function described here for human HP1 proteins is conserved. In Schizosaccharomyces pombe, the HP1 homologue, Swi6, is required to preserve genomic integrity and proper segregation of chromosomes during mitosis [34]. Impaired Swi6 function leads to mitotic alterations that cause severe growth alterations. Furthermore, the HP1-like protein in Dictyostelium discoideum, AX4 chromo domain-containing protein (hcpA), which displays $79 \%$ similarity to human HP1 $\gamma$, colocalizes with electron-dense structures at the nuclear periphery that are compatible with pericentrosomal material [35]. Overexpression of this protein causes growth defects that are accompanied by an increase in the frequency of atypical anaphase bridges. Genetic studies in Drosophila have demonstrated that mutations in the HP1 protein cause defective chromosome segregation [36,37]. Thus, in combination with this data, the studies described here indicate that HP1 proteins have evolved to support cell division in organisms ranging from fission yeast to humans.

Congruent with our results, previous experiments have defined a role for HP1 $\gamma$ in human diseases that are characterized by abnormal cell proliferation. High levels of HP1 1 have been observed in several cancer types, including esophageal, breast, colon, lung and cervical 
cancer, the cell model used here [10]. In addition, siRNA-mediated knockdown of $\mathrm{HP} 1 \gamma$ expression inhibits cervical cancer cell proliferation. Of note, Aurora A, the kinase identified in this study as responsible for $\mathrm{P}-\mathrm{Ser}^{83}-\mathrm{HP} 1 \gamma$ at $\mathrm{G}_{2} / \mathrm{M}$, is amplified and overexpressed in cervical cancer, which induces centrosome amplification, aneuploidy and transformation [38]. Cervical cancer patients with high Aurora A expression correlate with a poorer disease-free survival and overall survival rates than patients with low Aurora A expression, indicating that this protein could be used as a prognostic marker [39]. Based on the current study, the high levels of both HP1 $\gamma$ and Aurora kinases in cervical cancer cells would suggest that there is a resultant increase in P-Ser ${ }^{83}$-HP1 $\gamma$. Thus, targeting this pathway would affect P-Ser

${ }^{83}$-HP1 $\gamma$-mediated cell proliferation, in addition to other downstream Aurora effectors. In fact, Aurora kinase inhibitors have been shown to suppress proliferation of cervical cancer cells and enhance chemosensitivity $[40,41]$, suggesting that targeting Aurora in combination with the HP1-histone methyltransferase pathway may be a beneficial therapy in these patients.

\section{Conclusions}

In summary, the current study identifies a novel Aurora$\mathrm{HP} 1 \gamma$ pathway that involves $\mathrm{P}-\mathrm{Ser}^{83}-\mathrm{HP} 1 \gamma$ by Aurora A in $G_{2} / M$ and localization of this $\mathrm{HP} 1 \gamma$ subpopulation to the spindle pole, which is necessary for proper cell division. Combined, these results constitute robust evidence that P-Ser ${ }^{83}$-HP1 1 p plays a role in mitosis and bears importance for understanding impairments, which have been shown to be characterized by abnormally high levels of HP1 $\gamma$ and Aurora kinase activity, including cancer. Our results also suggest a teleological interpretation, namely that certain regulators of chromatin dynamics and transcription, such as HP1 $\gamma$, may undergo functional pressures (for example Aurora A phosphorylation) to maintain the integrity of cell division so that their own epigenetic inheritance is reproducible from cell generation to cell generation.

\section{Methods}

\section{Cell lines, reagents and cell treatments}

Cell lines were obtained from the American Type Culture Collection (ATCC, Rockville, MD, USA) and maintained according to the manufacturer's protocol. The human LX2 cell line was obtained as a generous gift from Dr Steve Freeman (Mount Sinai, NY, USA). Roscovitine (Sigma-Aldrich, St Louis, MO, USA) treatment was added at increasing concentrations $(0,5,10$ and $20 \mu \mathrm{M})$ for 8 hours, and lysates were harvested. Cells were treated with $3 \mu \mathrm{g} / \mathrm{ml}$ aphidicolin or $2 \mu \mathrm{g} / \mathrm{ml}$ nocodazole (both from EMD Millipore, Billerica, MA, USA) for 16 hours to arrest at $G_{1} / S$ and $G_{2} / M$, respectively. Control cells were treated with vehicle, dimethyl sulfoxide (DMSO). HeLa cells were synchronized by double thymidine block. Thymidine (2 mM, SigmaAldrich) was added to asynchronous cells for 18 hours. Cells were subsequently released for 9 hours in regular growth media prior to the second thymidine $(2 \mathrm{mM})$ block. After 17 hours, cells were released for the thymidine block and lysates were collected at the indicated time points. KT5720 was obtained from EMD Millipore. MLN8237 and hesperidin were purchased from Selleckchem (Houston, TX, USA). For hesperidin treatment, HeLa cells were arrested in mitosis by treatment with nocodazole for 16 hours. Arrested cells were treated with $200 \mathrm{nM}$ hesperidin for the indicated times in the presence of $10 \mu \mathrm{M}$ of the proteasome inhibitor MG132 (Sigma-Aldrich) to prevent mitotic exit [28].

\section{Plasmids, siRNA and recombinant adenovirus}

Standard molecular biology techniques were used to clone HP1 $\gamma$ into the pGEX and Ad5CMV vectors. For HP1 $\gamma$ specific transient shRNA-mediated knockdown, complementary oligonucleotides were synthesized for the target sequence (GCAAATCAAAGAAGAAAAG), annealed and ligated into the pCMS3 vector (kindly provided by Dr Daniel Billadeau, Mayo Clinic, Rochester, MN, USA). For stable shRNA-mediated HP1 $1 \gamma$ knockdown, control or HP1 $\gamma$-specific shRNA lentiviral particles (Santa Cruz Biotechnology, Inc, Santa Cruz, CA, USA) were used to infect cells according to the manufacturer's protocol, followed by puromycin selection $(2 \mu \mathrm{g} / \mathrm{ml})$. Myc-tagged wild type and dominant negative constructs for Aurora A and Aurora B were a kind gift from Dr Paolp Sassone-Corsi [21].

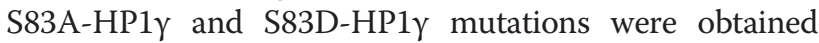
using the QuickChange Site-Directed Mutagenesis Kit, as suggested by the manufacturer (Agilent Technologies, Inc, Santa Clara, CA, USA). All constructs were verified by sequencing at the Molecular Biology Core at Mayo Clinic, Rochester, MN, USA. Aurora A $(A U R K A)$ and Aurora B $(A U R K B)$ Silencer validated siRNAs were purchased from Ambion-Life Technologies (Carlsbad, CA, USA). Epitopetagged (6xHis-Xpress) HP1 $\gamma$, S83A-HP1 $\gamma$ and S83D-HP1 $\gamma$ , as well as EV (Ad5CMV), were generated as recombinant adenovirus in collaboration with the Gene Transfer Vector Core at the University of Iowa, IA, USA.

\section{Western blot analysis}

Samples were run on 4 to 20\% gradient SDS-PAGE gels (Lonza, Walkersville, MD, USA) or 12\% SDS-PAGE gels and electroblotted onto polyvinylidene difluoride (PVDF) membranes (EMD Millipore). The membranes were blocked in 5\% BSA in tris-buffered saline Tween-20 (TBST) for 1 hour at room temperature. The blots were incubated for 2 hours at room temperature or overnight at $4^{\circ} \mathrm{C}$ with primary antibody (P-Ser ${ }^{83}-\mathrm{HP} 1 \gamma$ [8], 


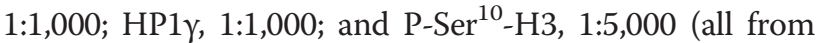
EMD Millipore); Aurora A, 1:1,000 (BD Biosciences Pharmingen, San Diego, CA, USA); Aurora B, 1:500; cyclin B1, 1:1,000; cyclin B2, 1:1,000; and CDK1, 1:1,000 (Abcam, Cambridge, MA, USA); $\beta$-actin, 1:1,000; and $\alpha$ tubulin, 1:1,000 (Sigma-Aldrich); c-Myc (9E10) for Myctagged proteins, 1:1,000 (Thermo Scientific, Rockford, IL, USA); and OMNI D8 for His-tagged proteins, 1:1,000 (Santa Cruz Biotechnology)). After repeated washes in TBST, horseradish peroxidase (HRP)-conjugated antirabbit or mouse IgG secondary antibody $(1: 5,000)$ was added for 1 hour at room temperature. Blots were developed by Pierce ECL chemiluminescent substrate (Thermo Scientific).

\section{Immunofluorescence and confocal microscopy}

Immunofluorescence and confocal microscopy were performed as previously described [42]. The primary antibodies were used at the following dilutions: P-Ser ${ }^{83}$ HP1 $\gamma$, 1:200; and $\gamma$-tubulin, 1:500 (Sigma-Aldrich); Aurora A, 1:50; and Aurora B, 1:50 (BD Biosciences Pharmingen); cyclin B1, 1:500; cyclin B2, 1:100; and CDK1, 1:40 (Abcam); and cyclin D3, 1:200 (Cell Signaling Technology, Danvers, MA, USA). For localization of P-Ser ${ }^{83}$-HP1 $\gamma$ during S-phase, EdU incorporation was combined with immunofluorescence. Prior to fixation, cells were incubated for 30 minutes in media containing 10 uM EdU. Subsequently, cells were processed for immunofluorescence, followed by EdU labeling using the Click-iT EdU Imaging Assay Kit (Invitrogen, Carlsbad, CA, USA) according to the manufacturer's protocol. For mitotic aberrations, spindle poles were labeled by immunofluorescence with $\gamma$-tubulin and counterstained with 4',6-diamidino-2-phenylindole (DAPI) containing mounting media (Vector Laboratories, Burlingame, CA, USA). For each condition, at least 200 mitotic cells were analyzed to quantify mitotic aberrations.

\section{GST fusion protein purification and in vitro kinase assays}

GST fusion protein purification was done as previously described [8]. For Aurora A and Aurora B in vitro kinase assays, HP1 fusion proteins $(10 \mu \mathrm{g})$ were incubated with recombinant kinases (EMD Millipore) and $10 \mathrm{mM}$ ATP (Sigma-Aldrich) for 10 minutes at $30^{\circ} \mathrm{C}$, in either the supplied buffer (Aurora A) or buffer containing $50 \mathrm{mM}$ Tris $\mathrm{pH} 7.5,0.1 \mathrm{mM}$ ethylene glycol tetraacetic acid (EGTA), and $15 \mathrm{mM}$ dithiothreitol (DTT, Aurora B). Kinase reactions were terminated by the addition of SDS loading dye and then resolved by western blot as described above.

\section{Cell proliferation assay}

Cell proliferation was measured by EdU incorporation using both fluorescence-activated cell sorting (FACS) and microscopy. Cells were infected with adenovirus

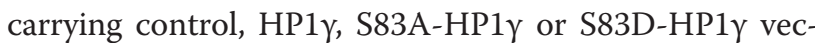
tors. Forty-eight hours post-plating, cells were pulsed with $10 \mu \mathrm{M}$ EdU (Invitrogen) for 1 hour. Subsequently, cells were processed using the Click-iT EdU Flow Cytometry or Imaging Assay Kits (Invitrogen) according to the manufacturer's protocols. EdU incorporation was measured by FACS analysis at the Mayo Flow Cytometry Research Core Facility, Rochester, MN, USA, or confocal microscopy. Each experiment was performed at least five different times in triplicate, expressed as means with standard error of mean (SEM) and statistical analyses were performed using unpaired $t$-test.

\section{Gene expression profiling, microarray analysis}

Global gene expression profiling was carried out at the Microarrays Facility of the Research Center of Laval University, CRCHUL, QC, Canada, utilizing the Affymetrix Human Gene 1.0 ST arrays (28,869 wellannotated genes and 764,885 distinct probes). Intensity files were generated by Affymetrix GCS 3000 7G and the GeneChip Operating Software (Affymetrix, Santa Clara, CA, USA). Data analysis, background subtraction and intensity normalization was performed using robust multi-array analysis (RMA) [43]. Genes that were differentially expressed along with false discovery rate were estimated from $t$-test $(>0.005)$ and corrected using Bayesian approach $[44,45]$. Data analysis, hierarchical clustering and ontology were performed with the oneChannelGUI to extend affylmGUI graphical interface capabilities [46], and Partek Genomics Suite, version 6.5 (Partek Inc, St Louis, MO, USA) with ANOVA analysis. Final fold changes were calculated as $\mathrm{x}=2^{\wedge} \log 2$ value. Probes with $P$ value $<0.05$ and fold change \pm 2.2 among

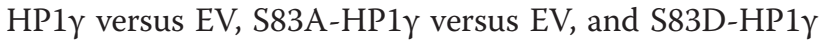
versus EV were selected for further analysis. For GO ANOVA, a minimum threshold of three genes and $P<0.05$ was used to identify significant functional groups. To validate the Affymetrix microarray, targets with significant alteration $(P<0.05)$ were compared to the realtime data using an arbitrary cutoff of \pm 2.2 fold change compared to EV control.

\section{Additional files}

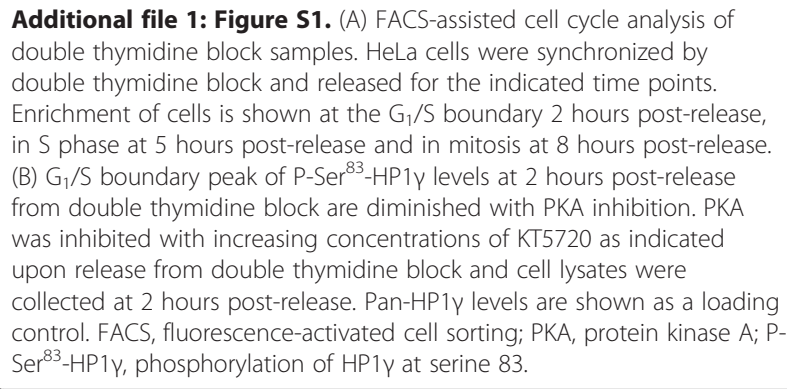

Additional file 1: Figure S1. (A) FACS-assisted cell cycle analysis of double thymidine block samples. HeLa cells were synchronized by double thymidine block and released for the indicated time points. Enrichment of cells is shown at the $G_{1} / S$ boundary 2 hours post-release, in $S$ phase at 5 hours post-release and in mitosis at 8 hours post-release (B) $G_{1} / S$ boundary peak of P-Ser ${ }^{83}-H P 1 \gamma$ levels at 2 hours post-release from double thymidine block are diminished with PKA inhibition. PKA was inhibited with increasing concentrations of KT5720 as indicated upon release from double thymidine block and cell lysates were collected at 2 hours post-release. Pan-HP1y levels are shown as a loading control. FACS, fluorescence-activated cell sorting; PKA, protein kinase A; P$\mathrm{Ser}^{83}-\mathrm{HP} 1 \gamma$, phosphorylation of HP1 $\gamma$ at serine 83. 
Additional file 2: Table S1. $q-P C R$ array validation of Affymetrix Human Gene 1.0 ST microarray.

\begin{abstract}
Abbreviations
ANOVA: analysis of variance; aph: aphidicolin; ATCC: American Type Culture Collection; BSA: bovine serum albumin; CDK: cyclin-dependent kinase; CDK1: cyclin-dependent kinase 1; CHO: Chinese hamster ovary; con: control; CRCHUL: Centre de Recherche du Centre Hospitalier de I'Université Laval; DAPI: 4',6-diamidino-2-phenylindole; DMSO: dimethyl sulfoxide; DTT: dithiothreitol; EdU: 5-ethynyl-2'-deoxyuridine; EGTA: ethylene glycol tetraacetic acid; EV: empty vector; FACS: fluorescence-activated cell sorting; GO: Gene Ontology; GST: glutathione S-transferase; GUI: graphical user interface; H3K9me: histone H3 lysine 9 methylation; HcpA: Dictyostelium discoideum, AX4 chromo domain-containing protein; HP1: heterochromatin protein 1; HRP: horseradish peroxidase; noc: nocodazole; Orc2: origin recognition complex subunit 2; PKA: protein kinase $\mathrm{A}_{\text {; }} \mathrm{P}_{-} \mathrm{Ser}^{10}{ }_{-}$ H3: phosphorylation of histone $\mathrm{H3}$ at serine 10; $\mathrm{P}-\mathrm{Ser}^{83}$ HP1Y: phosphorylation of HP1Y at serine 83; P-Thr ${ }^{288}$ : phosphorylation of Aurora A at threonine 288; PVDF: polyvinylidene difluoride; RMA: robust multi-array analysis; RT-PCR: reverse transcriptase polymerase chain reaction; SEM: standard error of mean; $\operatorname{Ser}^{10}$ : serine 10; $\operatorname{Ser}^{83}$ : serine 83; shCTRL: shRNA control; shHP1Y: shRNA knockdown of HP1 $\gamma$; shRNA: short hairpin RNA; siRNA: small interfering RNA; TBST: tris-buffered saline Tween-20.
\end{abstract}

\section{Competing interests}

The authors declare that they have no competing interests.

\section{Authors' contributions}

$\mathrm{RU}$ and $\mathrm{GL}$ generated the main idea of the work and developed the study design, both conceptually and methodologically. AG, PL, SS, AJM, GU, EC and GL made substantial contributions to the acquisition of data. AG, PL, EC, I, $\mathrm{RU}$ and $\mathrm{GL}$ contributed to analysis and interpretation of data. $\mathrm{AG}, \mathrm{RU}$ and $\mathrm{GL}$ wrote the manuscript from first draft to completion. AG, PL, SS, AJM, GU, EC, $J I, R U$ and $G L$ made comments, suggested appropriate modifications and corrections. All authors read and approved the final manuscript.

\section{Acknowledgements}

This work was supported by funding from the Fraternal Order of Eagles and a Career Development Award from the Mayo Clinic SPORE in Pancreatic Cancer (P50 CA102701, both to GL), as well as the National Institutes of Health (grant DK52913 to RU and T32CA148073 to AG), the Mayo Clinic Center for Cell Signaling in Gastroenterology (P30DK084567), and the Mayo Foundation. The authors would like to sincerely thank Dr Debora Bensi for technical assistance during the early development of this work, as well as Holger Dormann and $\operatorname{Dr} C$ David Allis for their generous contributions and helpful insights for the hesperidin P-Ser ${ }^{83}$-HP1Y experiments.

\section{Author details}

'Laboratory of Epigenetics and Chromatin Dynamics, GIH Division, Department of Medicine, Biochemistry and Molecular Biology, Guggenheim 10, Mayo Clinic, 200 First Street SW, Rochester, MN 55905, USA. ${ }^{2}$ Department of Obstetrics and Gynecology, Guggenheim 10, Mayo Clinic, 200 First Street SW, Rochester, MN 55905, USA. ${ }^{3}$ Molecular Endocrinology and Oncology Research Center, Centre Hospitalier de I'Universite Laval (CHUL) Research Center, Quebec, QC G1V 4G2, Canada. ${ }^{4}$ Institut National de la Santé et de la Recherche Médicale (INSERM), Unité 624, Stress Cellulaire, 163 Avenue de Luminy, Case 915, Parc Scientifique et Technologique de Luminy, 13288, Marseille Cedex 9, France. ${ }^{5}$ Translational Epigenomics Program, Center for Individualized Medicine, Rochester, MN 55905, USA.

Received: 14 February 2013 Accepted: 14 June 2013

Published: 5 July 2013

\section{References}

1. Eissenberg JC, James TC, Foster-Hartnett DM, Hartnett T, Ngan V, Elgin SC: Mutation in a heterochromatin-specific chromosomal protein is associated with suppression of position-effect variegation in Drosophila melanogaster. Proc Natl Acad Sci U S A 1990, 87:9923-9927.
2. James TC, Elgin SC: Identification of a nonhistone chromosomal protein associated with heterochromatin in Drosophila melanogaster and its gene. Mol Cell Biol 1986, 6:3862-3872.

3. Lomberk G, Wallrath L, Urrutia R: The Heterochromatin Protein 1 family. Genome Biol 2006, 7:228.

4. Kwon SH, Workman JL: The heterochromatin protein 1 (HP1) family: put away a bias toward HP1. Mol Cells 2008, 26:217-227.

5. Ayoub N, Jeyasekharan AD, Bernal JA, Venkitaraman AR: HP1-beta mobilization promotes chromatin changes that initiate the DNA damage response. Nature 2008, 453:682-686.

6. Martin C, Chen S, Heilos D, Sauer G, Hunt J, Shaw AG, Sims PFG, Jackson DA, Lovric J: Changed genome heterochromatinization upon prolonged activation of the Raf/ERK signaling pathway. PLoS One 2010, 5:e13322.

7. Zhao T, Heyduk T, Eissenberg JC: Phosphorylation site mutations in heterochromatin protein 1 (HP1) reduce or eliminate silencing activity. J Biol Chem 2001, 276:9512-9518.

8. Lomberk G, Bensi D, Fernandez-Zapico M, Urrutia R: Evidence for the existence of an HP1-mediated subcode within the histone code. Nat Cell Biol 2006, 8:407-415.

9. Maison C, Romeo K, Bailly D, Dubarry M, Quivy JP, Almouzni G: The SUMO protease SENP7 is a critical component to ensure HP1 enrichment at pericentric heterochromatin. Nat Struct Mol Biol 2012, 19:458-460.

10. Takanashi M, Oikawa K, Fujita K, Kudo M, Kinoshita M, Kuroda M: Heterochromatin protein 1 gamma epigenetically regulates cell differentiation and exhibits potential as a therapeutic target for various types of cancers. Am J Pathol 2009, 174:309-316.

11. Serrano A, Rodriguez-Corsino M, Losada A: Heterochromatin protein 1 (HP1) proteins do not drive pericentromeric cohesin enrichment in human cells. PLoS One 2009, 4:e5118.

12. Takada Y, Naruse C, Costa Y, Shirakawa T, Tachibana M, Sharif J, KezukaShiotani F, Kakiuchi D, Masumoto H, Shinkai Y, Ohbo K, Peters AH, Turner JM, Asano M, Koseki H: HP1gamma links histone methylation marks to meiotic synapsis in mice. Development 2011, 138:4207-4217.

13. Abe K, Naruse C, Kato T, Nishiuchi T, Saitou M, Asano M: Loss of heterochromatin protein 1 gamma reduces the number of primordial germ cells via impaired cell-cycle progression in mice. Biol Reprod 2011, 85:1013-1024.

14. Minc E, Allory Y, Worman HJ, Courvalin J-C, Buendia B: Localization and phosphorylation of HP1 proteins during the cell cycle in mammalian cells. Chromosoma 1999, 108:220-234.

15. Carmena M, Earnshaw WC: The cellular geography of Aurora kinases. Nat Rev Mol Cell Biol 2003, 4:842-854.

16. Meijer L, Borgne A, Mulner O, Chong JPJ, Blow JJ, Inagaki N, Inagaki M, Delcros JG, Moulinoux JP: Biochemical and cellular effects of roscovitine, a potent and selective inhibitor of the cyclin-dependent kinases cdc2, cdk2 and cdk5. Eur J Biochem 1997, 243:527-536.

17. Nigg EA: Mitotic kinases as regulators of cell division and its checkpoints. Nat Rev Mol Cell Biol 2001, 2:21-32.

18. Hendzel MJ, Wei Y, Mancini MA, Van Hooser A, Ranalli T, Brinkley BR, BazettJones DP, Allis CD: Mitosis-specific phosphorylation of histone H3 initiates primarily within pericentromeric heterochromatin during $\mathrm{G} 2$ and spreads in an ordered fashion coincident with mitotic chromosome condensation. Chromosoma 1997, 106:348-360.

19. Sardon T, Pache RA, Stein A, Molina H, Vernos I, Aloy P: Uncovering new substrates for Aurora A kinase. EMBO Rep 2010, 11:977-984.

20. Du J, Hannon GJ: Suppression of p160ROCK bypasses cell cycle arrest after Aurora-A/STK15 depletion. Proc Natl Acad Sci U S A 2004, 101:8975-8980.

21. Crosio C, Fimia GM, Loury R, Kimura M, Okano Y, Zhou H, Sen S, Allis CD, Sassone-Corsi P: Mitotic phosphorylation of histone H3: spatio-temporal regulation by mammalian Aurora kinases. Mol Cell Biol 2002, 22:874-885.

22. Li Z, Rana TM: A kinase inhibitor screen identifies small-molecule enhancers of reprogramming and iPS cell generation. Nat Commun 2012, 3:1085.

23. Murata-Hori M, Wang $Y L$ : The kinase activity of aurora $B$ is required for kinetochore-microtubule interactions during mitosis. Curr Biol 2002, 12:894-899.

24. Gorgun G, Calabrese E, Hideshima T, Ecsedy J, Perrone G, Mani M, Ikeda H, Bianchi G, Hu Y, Cirstea D, Santo L, Tai YT, Nahar S, Zheng M, Bandi M, Carrasco RD, Raje N, Munshi N, Richardson P, Anderson KC: A novel AuroraA kinase inhibitor MLN8237 induces cytotoxicity and cell-cycle arrest in multiple myeloma. Blood 2010, 115:5202-5213.

25. Asteriti I, Giubettini M, Lavia P, Guarguaglini G: Aurora-A inactivation causes mitotic spindle pole fragmentation by unbalancing microtubulegenerated forces. Mol Cancer 2011, 10:131. 
26. Hoar K, Chakravarty A, Rabino C, Wysong D, Bowman D, Roy N, Ecsedy J: MLN8054, a small-molecule inhibitor of Aurora A, causes spindle pole and chromosome congression defects leading to aneuploidy. Mol Cell Biol 2007, 27:4513-4525.

27. Gough NR: Focus issue: choreographing the dance of the mitotic kinases. Sci Signal 2011, 4:eg5.

28. Fischle W, Tseng BS, Dormann HL, Ueberheide BM, Garcia BA, Shabanowitz J, Hunt DF, Funabiki H, Allis CD: Regulation of HP1-chromatin binding by histone H3 methylation and phosphorylation. Nature 2005, 438:1116-1122.

29. Hirota T, Lipp JJ, Toh BH, Peters JM: Histone H3 serine 10 phosphorylation by Aurora B causes HP1 dissociation from heterochromatin. Nature 2005, 438:1176-1180.

30. Prasanth SG, Prasanth KV, Siddiqui K, Spector DL, Stillman B: Human Orc2 localizes to centrosomes, centromeres and heterochromatin during chromosome inheritance. EMBO J 2004, 23:2651-2663.

31. Kondo $Y$, Shen L, Ahmed S, Boumber Y, Sekido Y, Haddad BR, Issa JP. Downregulation of histone $\mathrm{H} 3$ lysine 9 methyltransferase G9a induces centrosome disruption and chromosome instability in cancer cells. PLOS One 2008, 3:e2037.

32. Chin HG, Esteve P-O, Pradhan M, Benner J, Patnaik D, Carey MF, Pradhan S: Automethylation of G9a and its implication in wider substrate specificity and HP1 binding. Nucleic Acids Res 2007, 35:7313-7323.

33. Ogawa H, Ishiguro K, Gaubatz S, Livingston DM, Nakatani Y: A complex with chromatin modifiers that occupies E2F- and Myc-responsive genes in G0 Cells. Science 2002, 296:1132-1136.

34. Nonaka N, Kitajima T, Yokobayashi S, Xiao G, Yamamoto M, Grewal SIS, Watanabe $Y$ : Recruitment of cohesin to heterochromatic regions by Swi6/HP1 in fission yeast. Nat Cell Biol 2002, 4:89-93.

35. Kaller M, Euteneuer U, Nellen W: Differential effects of heterochromatin protein 1 isoforms on mitotic chromosome distribution and growth in Dictyostelium discoideum. Eukaryot Cell 2006, 5:530-543.

36. Fanti L, Giovinazzo G, Berloco M, Pimpinelli S: The heterochromatin protein 1 prevents telomere fusions in Drosophila. Mol Cell 1998, 2:527-538.

37. Kellum R, Alberts BM: Heterochromatin protein 1 is required for correct chromosome segregation in Drosophila embryos. J Cell Sci 1995, 108:1419-1431.

38. Zhou H, Kuang J, Zhong L, Kuo WL, Gray JW, Sahin A, Brinkley B, Sen S: Tumour amplified kinase STK15/BTAK induces centrosome amplification, aneuploidy and transformation. Nat Genet 1998, 20:189-193.

39. Zhang W, Wang J, Liu SJ, Hua W, Xin XY: Correlation between Aurora-A expression and the prognosis of cervical carcinoma patients. Acta Obstet Gynecol Scand 2009, 88:521-527.

40. Cheung CH, Lin WH, Hsu JT, Hour TC, Yeh TK, Ko S, Lien TW, Coumar MS Liu JF, Lai WY, Shiao HY, Lee TR, Hsieh HP, Chang JY: BPR1K653, a novel Aurora kinase inhibitor, exhibits potent anti-proliferative activity in MDR1 (P-gp170)-mediated multidrug-resistant cancer cells. PLoS One 2011, 6:e23485.

41. Zhang L, Zhang S: ZM447439, the Aurora kinase B inhibitor, suppresses the growth of cervical cancer SiHa cells and enhances the chemosensitivity to cisplatin. J Obstet Gynaecol Res 2011, 37:591-600.

42. Gebelein B, Urrutia R: Sequence-specific transcriptional repression by KS1, a multiple-zinc-finger-Kruppel-associated box protein. Mol Cell Biol 2001, 21:928-939.

43. Irizarry RA, Hobbs B, Collin F, Beazer-Barclay YD, Antonellis KJ, Scherf U, Speed TP: Exploration, normalization, and summaries of high density oligonucleotide array probe level data. Biostatistics 2003, 4:249-264.

44. Benjamini Y, Drai D, Elmer G, Kafkafi N, Golani I: Controlling the false discovery rate in behavior genetics research. Behav Brain Res 2001, 125:279-284.

45. Smyth GK: Linear models and empirical bayes methods for assessing differential expression in microarray experiments. Stat Appl Genet Mol Biol: Stat Appl Genet Mol Biol; 2004:3. Article3.

46. Wettenhall JM, Simpson KM, Satterley K, Smyth GK: affylmGUI: a graphical user interface for linear modeling of single channel microarray data. Bioinformatics 2006, 22:897-899.

doi:10.1186/1756-8935-6-21

Cite this article as: Grzenda et al:: Functional impact of Aurora Amediated phosphorylation of HP1 1 at serine 83 during cell cycle progression. Epigenetics \& Chromatin 2013 6:21.

\section{Submit your next manuscript to BioMed Central and take full advantage of:}

- Convenient online submission

- Thorough peer review

- No space constraints or color figure charges

- Immediate publication on acceptance

- Inclusion in PubMed, CAS, Scopus and Google Scholar

- Research which is freely available for redistribution 